\title{
Long-Term Characterization of Hippocampal Remapping during Contextual Fear Acquisition and Extinction
}

\author{
Peter J. Schuette, ${ }^{1 \star}$ Fernando M. C. V. Reis, ${ }^{1 \star}$ Sandra Maesta-Pereira, ${ }^{1}$ Meghmik Chakerian, ${ }^{1}$ Anita Torossian, ${ }^{1}$ \\ ${ }^{\circledR}$ Garrett J. Blair, ${ }^{1}$ Weisheng Wang, ${ }^{1}$ Hugh T. Blair, ${ }^{1}$ Michael S. Fanselow, ${ }^{1}$ Jonathan C. Kao, ${ }^{2 \star}$ and \\ Avishek Adhikari ${ }^{1 *}$ \\ ${ }^{1}$ Department of Psychology, University of California, Los Angeles, Los Angeles, California 90095, and ${ }^{2}$ Department of Electrical and Computer \\ Engineering, University of California, Los Angeles, Los Angeles, California 90095
}

\begin{abstract}
Hippocampal CA1 place cell spatial maps are known to alter their firing properties in response to contextual fear conditioning, a process called "remapping." In the present study, we use chronic calcium imaging to examine remapping during fear retrieval and extinction of an inhibitory avoidance task in mice of both sexes over an extended period of time and with thousands of neurons. We demonstrate that hippocampal ensembles encode space at a finer scale following fear memory acquisition. This effect is strongest near the shock grid. We also characterize the long-term effects of shock on place cell ensemble stability, demonstrating that shock delivery induces several days of high fear and low between-session place field stability, followed by a new, stable spatial representation that appears after fear extinction. Finally, we identify a novel group of CA1 neurons that robustly encode freeze behavior independently from spatial location. Thus, following fear acquisition, hippocampal CA1 place cells sharpen their spatial tuning and dynamically change spatial encoding stability throughout fear learning and extinction.
\end{abstract}

Key words: calcium imaging; contextual fear conditioning; hippocampus; miniaturized microscope; place cell; remapping

Significance Statement

The hippocampus contains place cells that encode an animal's location. This spatial code updates, or remaps, in response to environmental change. It is known that contextual fear can induce such remapping; in the present study, we use chronic calcium imaging to examine inhibitory avoidance-induced remapping over an extended period of time and with thousands of neurons and demonstrate that hippocampal ensembles encode space at a finer scale following electric shock, an effect which is enhanced by threat proximity. We also identify a novel group of freeze behavior-activated neurons. These results suggest that, more than merely shuffling their spatial code following threat exposure, place cells enhance their spatial coding with the possible benefit of improved threat localization.

\section{Introduction}

The hippocampal CA1 region contains neurons that fire in response to specific locations of an environment (O'Keefe and Dostrovsky, 1971), which are often stable across days (Muller and

Received Apr. 28, 2020; revised Sep. 3, 2020; accepted Sep. 9, 2020.

Author contributions: F.M.C.V.R., S.M.-P., M.C., A.T., G.J.B., and W.W. performed research; F.M.C.V.R., H.T.B., M.S.F., and A.A. designed research; H.T.B. contributed unpublished reagents/analytic tools; P.J.S., F.M.C.V.R., and J.C.K. analyzed data; P.J.S., M.S.F., and A.A. wrote the paper.

*P.J.S., F.M.C.V.R., J.C.K., and A.A. contributed equally to this work.

This work was supported by National Institute for Mental Health Grants R00 MH106649 and R01 MH119089 (to A.A.), Brain \& Behavior Research Foundation Grants 22663, 27654, and 27780 (respectively, to A.A, F.M.C.V.R. and W.W), the National Science Foundation Grant NSF-GRFP DGE-1650604 (to P.J.S.), the University of California, Los Angeles Affiliates Fellowship (P.J.S.), the Hellman Foundation (A.A.), and the Fundação de Amparo à Pesquisa do Estado de São Paulo (FAPESP) Research Grant 2014/05432-9. F.M.C.V.R. was supported by FAPESP Grants 2015/23092-3 and 2017/08668-1.

The authors declare no competing financial interests.

Correspondence should be addressed to Avishek Adhikari at avi@psych.ucla.edu.

https://doi.org/10.1523/JNEUROSCI.1022-20.2020

Copyright $\odot 2020$ the authors
Kubie, 1987; Thompson and Best, 1990; Wilson and McNaughton, 1993; Kinsky et al., 2018; Gonzalez et al., 2019). It is thought that place cells may assist in the recognition of, and discrimination between, different environmental contexts (Phillips and LeDoux, 1994; Frankland et al., 1998; Latuske et al., 2018).

Place cell ensembles have been shown to update, or remap, their firing properties based on both changes in the physical layout of the environment and other salient features. Remapping can occur on a global scale, such that place cell ensembles premanipulation and postmanipulation are orthogonal (O'Keefe and Conway, 1978; Muller and Kubie, 1987; Thompson and Best, 1989; Kubie and Muller, 1991; Leutgeb et al., 2005b; Alme et al., 2014). Large manipulations are required to induce global remapping, such as a drastic change of enclosure shape or distal cues. Remapping may also occur on a partial scale and appear as an incomplete change in place cell firing maps (spatial remapping) and/or firing rate (rate remapping). This could result from a change in the color or odor of an enclosure (Anderson and 
Jeffery, 2003; Wood et al., 1999), or a more incremental change of enclosure shape (Leutgeb et al., 2005a). This process may allow for the independent hippocampal representation of spatial position and other salient contextual cues.

Threat exposure may also cause both spatial and rate remapping; such changes have been induced by electric shock (Moita et al., 2004; Wu et al., 2017), a predatory robot (Kim et al., 2015), and exposure to predator urine (Wang et al., 2012, 2015). However, several important questions remain unanswered, despite the pioneering contributions of these works. How does the activity of the same cells change during fear acquisition and extinction remapping over the course of a long period of time? Do hippocampal cells encode fear-induced behaviors such as freezing? Does spatial information content change following fear acquisition in a way that reflects the place cell's distance to the threat? To address these issues, we obtained chronic recordings of large CA1 ensembles, allowing us to track how activity of the same cells changes during fear acquisition and extinction across a span of multiple days.

\section{Materials and Methods}

\section{Subjects}

All procedures conformed to guidelines established by the National Institutes of Health and have been approved by the University of California, Los Angeles Institutional Animal Care.

\section{Mice}

C57BL/6N mice were used for all experiments. Male and female mice between four and six months of age were used in all experiments. Mice were maintained on a 12 -h reverse light-dark cycle with food and water ad libitum. All mice were handled for a minimum of $5 \mathrm{~d}$ before any behavioral task.

\section{Viral vectors}

AAV9.Syn.GCaMP6f.WPRE.SV40 virus (titer: $1.40 \times 1013 \mathrm{GC} / \mathrm{ml}$ ) was purchased from Penn Vector Core.

\section{Surgeries}

Surgical methods were similar to those reported elsewhere (Cai et al., 2016). Briefly, mice were unilaterally injected with $500 \mathrm{nl}$ of AAV9.Syn. GCaMP6f.WPRE.SV40 virus at $60 \mathrm{nl} / \mathrm{min}$ into the dorsal CA1 at the stereotactic coordinates $-2.1 \mathrm{~mm}$ posterior to bregma, $1.6 \mathrm{~mm}$ lateral to midline, and $-1.60 \mathrm{~mm}$ ventral to skull surface. Following $5 \mathrm{~d}$ of recovery, a microendoscope was implanted above the injection site. The microendoscope (GRIN lens, 0.25 pitch, $0.50 \mathrm{NA}, 1.8 \mathrm{~mm}$ in diameter, Edmund Optics) was placed above the CA1 region of the hippocampus by stereotaxic arm at a depth of $1.35 \mathrm{~mm}$, ventral to the skull surface. Two skull screws were placed and the lens was secured with cyanoacrylate glue and dental cement. The exposed end of the GRIN lens was protected with transparent Kwik-seal glue and animals were returned to a clean cage. Two weeks later, a small aluminum base plate was cemented onto the animal's head atop the previously formed dental cement.

\section{Behavior and miniscope video capture}

All videos were recorded at 30 frames/s using a Logitech HD C310 webcam and custom-built head-mounted UCLA miniscope (Aharoni and Hoogland, 2019). Open-source UCLA Miniscope software and hardware (http://miniscope.org/) were used to capture and synchronize neural and behavioral video.

\section{Perfusion and histologic verification}

Mice were anesthetized with Fatal-Plus and transcardially perfused with phosphate buffered saline followed by a solution of $4 \%$ paraformaldehyde. Extracted brains were stored for $12 \mathrm{~h}$ at $4^{\circ} \mathrm{C}$ in $4 \%$ paraformaldehyde. Brains were then placed in sucrose for a minimum of $24 \mathrm{~h}$. Brains were sectioned in the coronal plane in a cryostat, washed in phosphate buffered saline and mounted on glass slides using PVA-DABCO. Images were acquired using a Keyence BZ-X fluorescence microscope with a $10 \times$ or $20 \times$ air objective.

\section{Experimental design and statistical analysis}

Full grid assay

All measurements are written as length $\times$ width $\times$ height in $\mathrm{cm}$. Mice were placed in an empty open field $(63 \times 10 \times 40 \mathrm{~cm})$ for a period of $10 \mathrm{~min}$. To encourage the formation of stable place fields, local cues were provided in the form of colored tape along the walls of the enclosure. Four separately controllable shock grids were positioned across the length of the floor. On the seventh recording day, a series of 102 -s 0.4 $\mathrm{mA}$ footshocks were delivered at pseudo-random intervals to the occupied shock grid. The first five shocks were exclusively delivered on the corner grids. The sixth, seventh and eighth shocks were delivered on the middle grids. The last two shocks were delivered on the corner grids. So that all shocks were delivered, the total duration of these sessions varied from 10 to $12 \mathrm{~min}$. This procedure was used so that the animals received shocks throughout the enclosure, associating the entire environment, rather than a subsection, with shocks. For better spatial sampling, shocks were preferentially targeted to the corner grids, as mice innately prefer these areas. By rendering the corners less safe, we aimed to encourage exploration of the entire enclosure. Recording then continued, without shock, for six more days for 10-min sessions each. A total of six mice were used ( $n=6$ males).

\section{Local grid assay}

Mice were placed in an empty open field $(63 \times 10 \times 40 \mathrm{~cm})$ for a period of $10 \mathrm{~min}$. Similar to the full grid assay, local cues were provided in the form of colored tape along the walls of the enclosure. A single shock grid was placed at the extreme end of the enclosure. On the seventh recording day, the grid was activated, such that a $0.4-\mathrm{mA}$ footshock was delivered for $2 \mathrm{~s}$ whenever the mouse fully entered the grid zone. Recording then continued, without shock, for nine more days. A total of eight mice were analyzed ( $n=5$ males; $n=3$ females). One mouse was excluded because of insufficient avoidance of the shock grid following shock delivery.

\section{Control (no-shock) assay}

The control assay was identical to the full and local grid assays, with the exception that no shock grids were used. A total of four mice were used ( $n=4$ males).

\section{Behavioral quantification}

To extract the pose of freely-behaving mice in the described essays, we implemented DeepLabCut (Mathis et al., 2018), an open-source convolutional neural network-based toolbox, to identify mouse nose, ear and tailbase $x y$-coordinates in each recorded video frame. These coordinates were then used to calculate speed and position at each time point, as well as classify freezing behavior in an automated manner using custom MATLAB scripts. Freezing was defined as epochs when head and tailbase velocities fall below $0.25 \mathrm{~cm} / \mathrm{s}$ for a period of at least $0.33 \mathrm{~s}$. The durations of all freezing episodes within a session were summed to calculate the total freeze duration. Stretch-attend posture was defined as epochs when the distance from mouse nose to tail base exceeded a minimum threshold for a period of at least $0.5 \mathrm{~s}$. Locomotion bouts were defined as periods in which head speed exceeded $1 \mathrm{~cm} / \mathrm{s}$ for a period of at least $0.5 \mathrm{~s}$, and the beginning and end head locations were $>2 \mathrm{~cm}$ apart.

\section{Miniscope postprocessing and coregistration}

Miniscope videos were motion-corrected using the open-source UCLA miniscope analysis package (https://github.com/daharoni/Miniscope Analysis; Aharoni et al., 2019). They were spatially downsampled by a factor of two and temporally downsampled by a factor of four, and the cell footprints and activity were extracted and calcium events deconvolved using the open-source package Constrained Nonnegative Matrix Factorization for microEndoscopic data (CNMF-E; https://github.com/ zhoupc/CNMF_E; Zhou et al., 2018). Neurons were coregistered across 
A

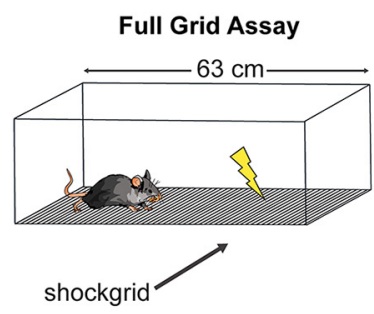

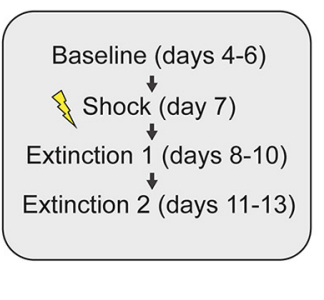

D

C
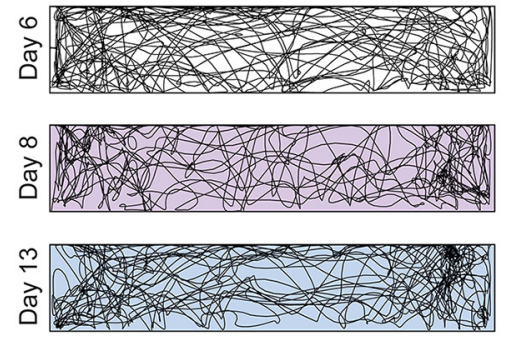

$\mathbf{F}$

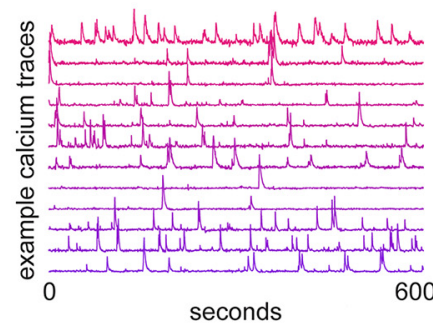

G

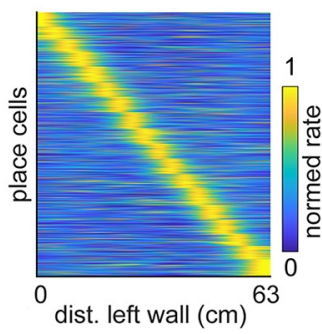

B

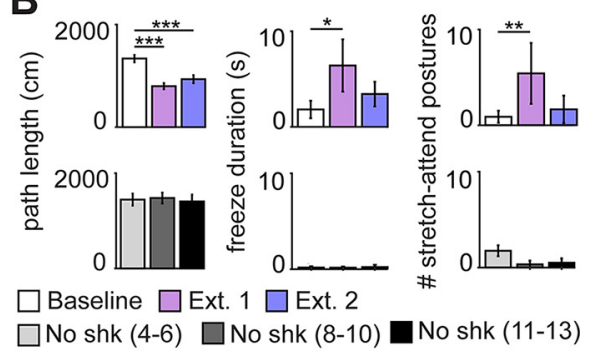

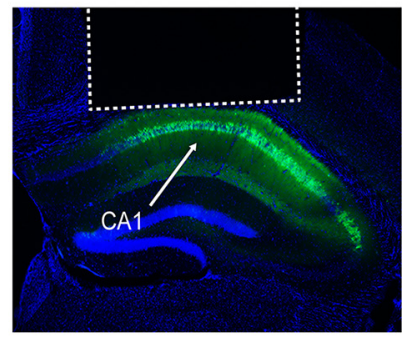

E

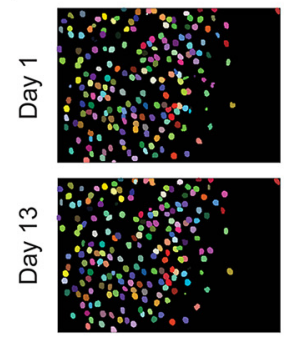

H

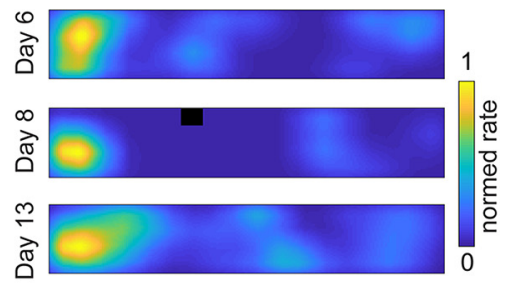

Figure 1. Experimental design, example data and analysis for full grid assay. $\boldsymbol{A}$, Illustration of full grid inhibitory avoidance assay and epoch sequence. $\boldsymbol{B}$, Mean path length (left), freeze duration (middle), and number of stretch-attend postures (right) of mice across recording epochs ( \pm 1 SEM) for (top) full grid assay and (bottom) control no shock (No shk) assay ( $n=6$ full grid; $n=4$ control). Statistical comparisons are performed by Wilcoxon rank-sum test for baseline/ext. 1 and baseline/ext. 2 sessions, as well as for matched sessions of the control assay. $C$, Example paths from day 6 (baseline), day 8 (extinction epoch 1), and day 13 (extinction epoch 2). D, Example injection site, showing lens placement (dotted line) and expression of GCaMP6f in the hippocampal CA1 region. $\boldsymbol{E}$, Example imaging field of view with cell bodies, color coded by coregistration procedure across the first and last day of recording (days 1 and 13 , respectively). $\boldsymbol{F}$, Example raw calcium traces from a subset of cells recorded during a single session. $\mathbf{G}$, Rows depict the rate maps of all place cell instances in all full grid sessions along the length of the full grid enclosure, sorted by place field center location relative to distance from the left wall $(n=17,051)$. $\boldsymbol{H}$, Normalized rate maps of an example place cell, stable across recording epochs; ${ }^{*} p<0.05,{ }^{* *} p<0.01,{ }^{* * *} p<0.001$.

sessions using the open-source probabilistic modeling package CellReg (https://github.com/zivlab/CellReg; Sheintuch et al., 2017).

\section{Place maps}

Given that calcium events are indirect estimates of neural activity, we inferred spike times through deconvolution. Inferred spikes, hereafter referred to as "calcium events," were deconvolved from calcium activity using the OASIS algorithm (Friedrich et al., 2017) included with CNMFE. Deconvolved calcium events were spatially binned into $3 \times 3 \mathrm{~cm}$ pixels and normalized by total occupancy, generating a rate map, or the event rate of each neuron at each binned location. Bins occupied for less than five samples were excluded from the analysis, as were periods of freeze behavior. To control for the possible behavioral confound of decreased postshock speed, we included only samples for which mouse speed was between 0.5 and $2.6 \mathrm{~cm} / \mathrm{s}$, as the mean speed and number of included samples within this range was not significantly different between baseline and extinction epochs. Rate maps were smoothed by convolution with a Gaussian kernel $(5 \times 5$ bins, $1 \mathrm{SD}=0.85$ bins or $2.55 \mathrm{~cm})$.

Place cell properties

Only samples with speed between 0.5 and $2.6 \mathrm{~cm} / \mathrm{s}$ were used to calculate the following place cell measures (see above, Place maps). Mean event rate was defined as the total number of calcium events divided by the number of behavioral samples. Place field size was measured by the number of rate map bins contiguous with the peak rate bin and exceeding the mean rate by $>1.5 \mathrm{SD}$.

A standard information-theoretic approach (Skaggs et al., 1993) was applied to the rate map of each cell to determine its spatial information content in bits/event. To be classified as a place cell, a neuron had to (1) exceed 0.2 bits/event and (2) exhibit more than five calcium events over the course of the session. Moreover, the information content of each place cell had to (3) exceed $95 \%$ of a bootstrapped distribution. To build this distribution, calcium event times were randomized and the spatial information content was calculated over 1000 iterations for each cell.

We performed two analyses on the full grid dataset to address the concern that unequal spatial sampling, preshock and postshock, could potentially drive our results:

(1) We recalculated our main place field firing properties on a subsampled dataset, for which an equal number of samples was included from the left and right sides of the enclosure for each session.

(2) We used the binned occupancy map along the length of the enclosure to calculate Sarle's bimodality coefficient (Ellison, 1987), the degree of bimodality within each session:

$$
\text { Bimodality coefficient }=\left(\text { skewness }^{2}+1\right) / \text { kurtosis },
$$

where skewness and kurtosis are the third and fourth standardized moment of the distribution, respectively. 


$$
\begin{aligned}
& \text { skewness }=\mathrm{E}\left(([X-\mu] / \sigma)^{\wedge} 3\right) \\
& \text { kurtosis }=\mathrm{E}\left(([X-\mu] / \sigma)^{\wedge} 4\right) .
\end{aligned}
$$

We then calculated the Spearman correlation between these values and the mean event rate, place field size, and spatial information content separately for all preshock and postshock sessions.

\section{Place cell property cross-epoch comparison}

To statistically compare place cell firing properties between baseline and extinction (Ext) epochs, all place cells that coregistered between baseline sessions 4 and 6 and Ext sessions 8 and 10, 11 and 13, or 14 and 16 (local grid only) were considered. For cells that coregistered across more than one baseline or Ext session, only the values for sessions closest in time were considered. When comparing place field firing properties between the left and right sides of the local grid and control assays, the same closest-in-time criterion was used. Moreover, to be included in the left/right analysis, place cells had to maintain their place field centers on either the left or right side of the enclosure from baseline to Ext.

\section{Classification of stable place cells}

A place cell was considered stable across contiguous sessions if it both fulfilled place cell criteria for neighboring sessions and exhibited a place field center shift of $<5 \mathrm{~cm}$.

\section{Rate map correlation analysis}

To determine the place field similarity between sessions, the rate maps of all coregistered place cells were considered. The Pearson correlation $(r)$ was calculated for corresponding rate map bins across all mice. If a bin had inadequate sampling (less than five samples) in either session, it was excluded from the correlation analysis.

\section{Freeze cell classification}

To classify CA1 cells as freeze-encoding, we used a generalized linear model (GLM) to identify cells that showed increases in calcium activity during freeze behavior. The model was fit to the activity of each cell, with freeze indices as the predictor variable and freeze coefficient as the measure of fit. Freeze onset times were consistently rolled by multiples of 2-s intervals and a bootstrap distribution built from the resulting GLM coefficients. A cell was considered a freeze cell if its coefficient exceeded $95 \%$ of the bootstrap coefficient values. We chose to train the GLM on $\mathrm{dF} / \mathrm{F}$, rather than deconvolved activity, because (1) the slow decay of the calcium signal allows for neural activity preceding freeze behavior to contribute toward the linear model of that behavior, (2) there is a convention to do so in the calcium imaging literature (Engelhard et al., 2019; Pinto et al., 2019), and (3) using the calcium activity directly, we avoid any underlying assumptions of the deconvolution procedure.

Percent freeze cells activated during freeze behavior at multiple locations To calculate this percentage, freeze bouts were categorized by the quarter of the enclosure in which they occurred. Only sessions for which freeze occurred at multiple locations (fraction of total freeze bouts to occur at freeze location mode $<0.7$ ) were considered. Moreover, only freeze cells active for at least three freeze events were included in the analysis. If a

A

E

G

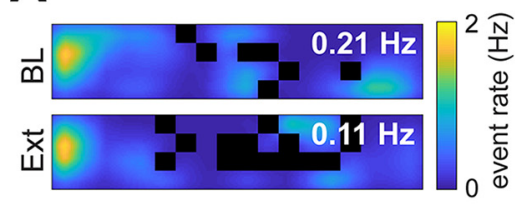

B
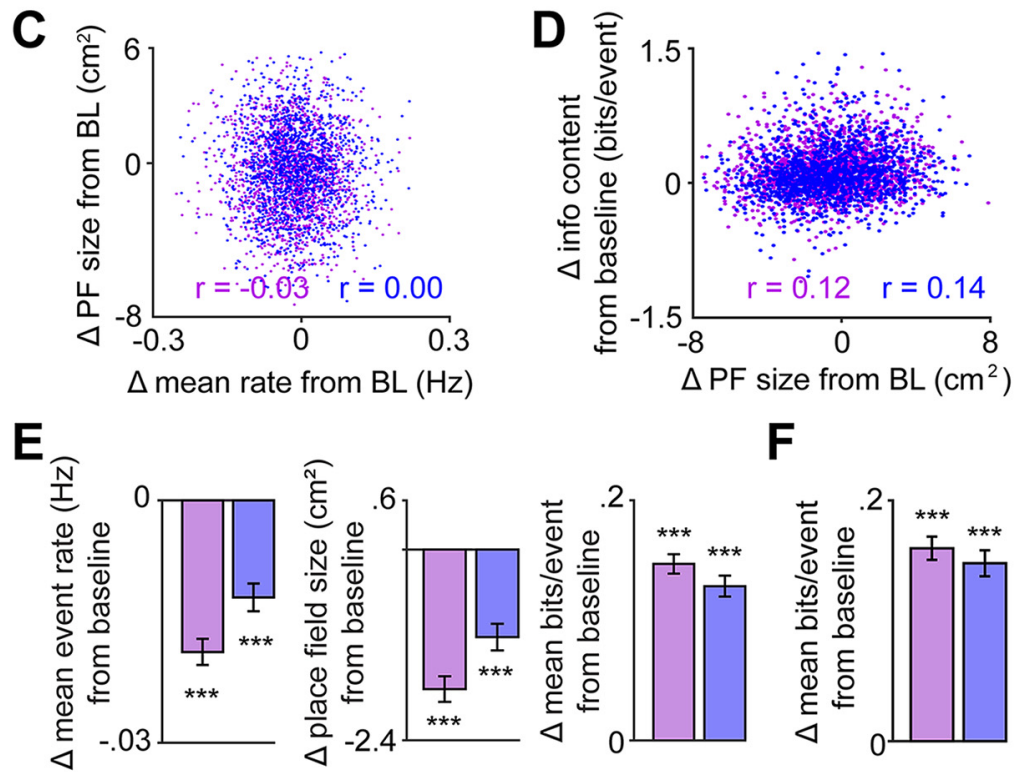

$\mathbf{F}$
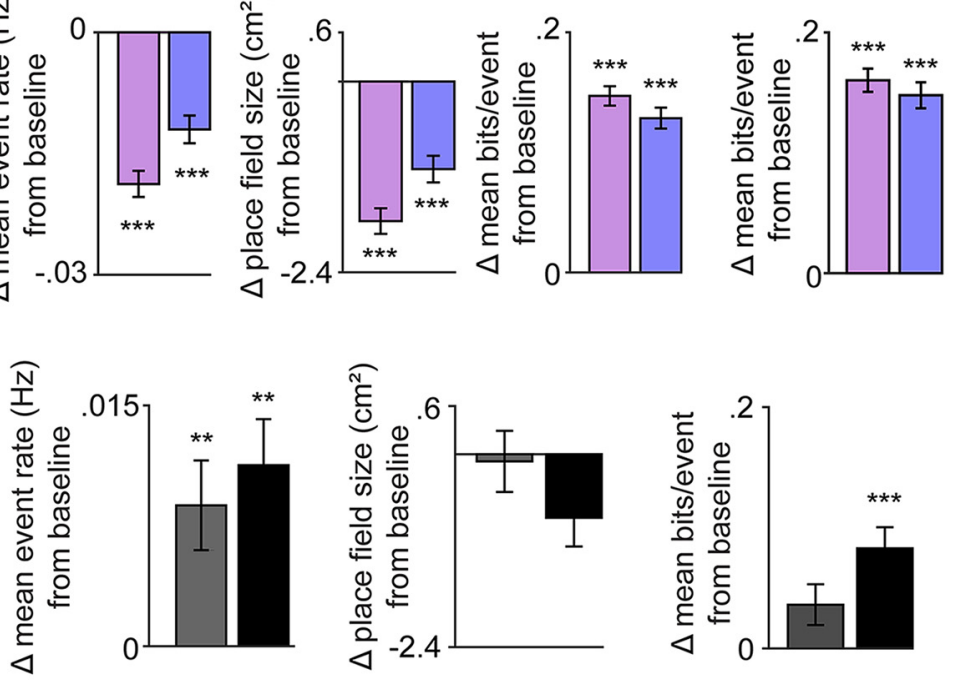

$\square$ Ext. 1 (days 8-10)
$\square$ No shock (days 8-10)

Ext. 2 (days 11-13)

No shock (days 11-13)

Figure 2. Fear acquisition decreases mean rate, place field size and increases information content. $\boldsymbol{A}$, Example rate maps of place cell with a decrease in mean rate from baseline to extinction (Ext). $\boldsymbol{B}$, Example rate maps of place cell with a decrease in field size from baseline to Ext. $\boldsymbol{C}$, Correlation of change in field size and change in mean rate from baseline to Ext 1 and 2 ( $n=1620$ Ext 1, $n=1535$ Ext 2; baseline/Ext 1 Spearman $r=-0.03, p>0.05$; baseline/Ext 2 Spearman $r=0.00, p>0.05)$. $\boldsymbol{D}$, Correlation of the change in spatial information content and place field size from baseline $(n=1620$ Ext 1, $n=1535$ Ext 2; baseline/Ext 1 Spearman $r=0.12, p<0.001$; baseline/Ext 2 Spearman $r=0.14$, $p<0.001)$. For $\boldsymbol{E}-\boldsymbol{G}$, statistical comparisons are performed by Wilcoxon signed-rank test for paired cells at baseline/Ext 1 and baseline/Ext 2 (see Materials and Methods). $\boldsymbol{E}$, Changes in place cell firing properties from baseline across epochs of the full grid assay. Bars show the changes in firing properties ( \pm 1 SEM) of pooled place cells that coregister between baseline and Ext epochs 1 and 2 ( $n=1620$ baseline/Ext $1 ; n=1535$ baseline/Ext 2). $\boldsymbol{F}$, Change in spatial information content ( \pm 1 SEM) from baseline to extinction 1 and 2 epochs, recalculated for only place cells that did not show a concurrent decrease in place field size ( $n=793$ baseline/Ext $1 ; n=748$ baseline/Ext 2). $\mathbf{G}$, Changes in firing properties of hippocampal CA1 place cells across epochs of control (no shock) assay. Bars show the changes in firing properties $( \pm 1$ SEM) of pooled cells that coregister between no-shock days 4 and 6 and days 8 and 10 and 11 and 13 ( $n=302$, days $4-10 /$ days $8-10 ; n=304$, days $4-10 /$ days $11-13) ;{ }^{* *} p<0.01,{ }^{* * *} p<0.001$.

freeze cell was active within $\pm 1 \mathrm{~s}$ of freeze onset in at least two different quarters of the enclosure for a given session, it contributed to this percentage.

Putative sharp wave ripple ( $p S W R$ ) classification

Multiunit activity was used to define pSWRs as any sample whose calcium event count exceeded the mean by $>3$ SDs (Davidson et al., 2009). 
A

Metrics calculated with equal sampling from left and right sides of enclosure
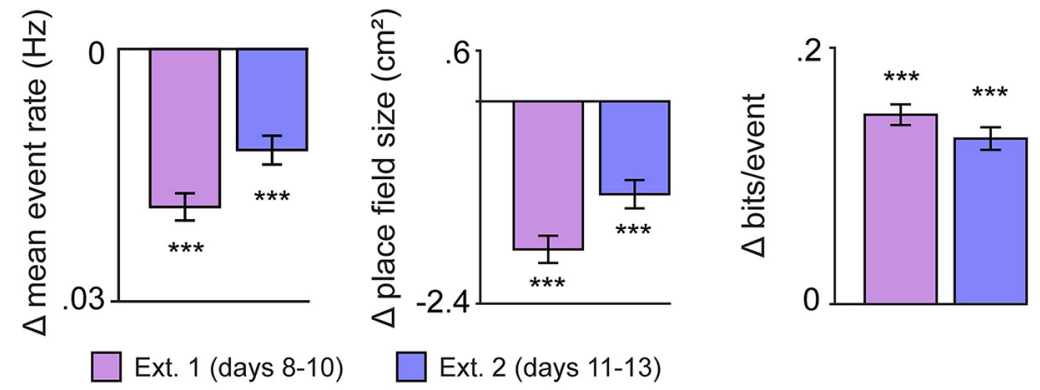

B

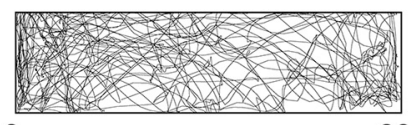

0

63

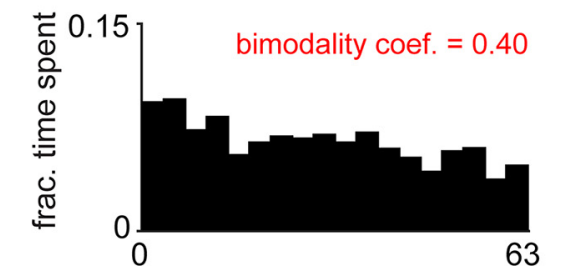

0.15

0
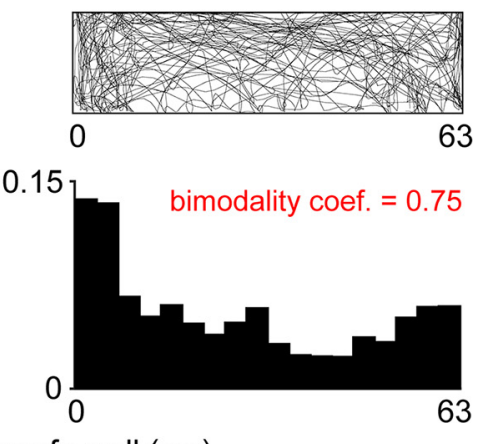

distance from safe wall $(\mathrm{cm})$

C
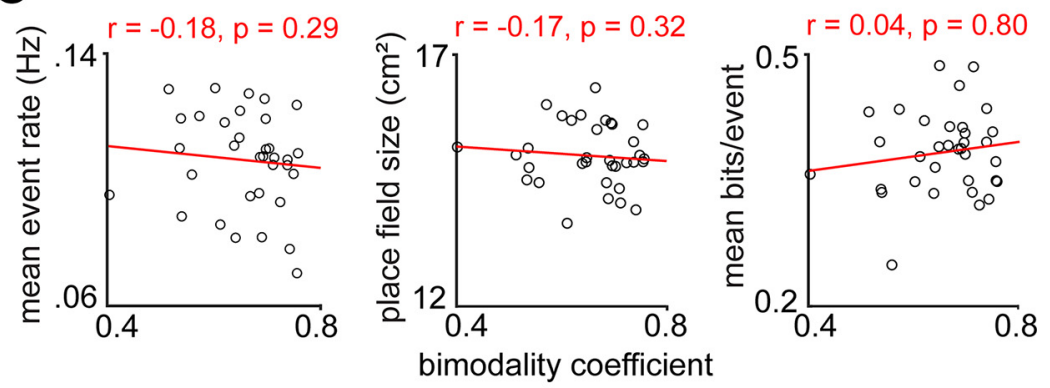

Values from baseline session (days 1-6)

Figure 3. Effects of behavioral sampling on place cell firing properties. $A$, Bars depict the same place cell firing properties ( \pm 1 SEM) described in Figure $2 E$, but resampled such that, for each session, there is an equal number of samples from the left and right side of the enclosure ( $n=1620$ baseline/Ext $1 ; n=1535$ baseline/Ext 2). $\boldsymbol{B}$, top, Lines depict example tracks from preshock and postshock sessions. Bottom, Bars show the fraction of time the mouse spent at binned positions along the length of the enclosure, with the corresponding bimodality coefficient in red. $\boldsymbol{C}$, The correlation of the bimodality coefficient with mean event rate, place field size and information content for all preshock sessions $(n=36$ preshock sessions); ${ }^{* * *} p<0.001$.

\section{Statistics}

Significance values are included in the figure legends. Unless otherwise noted, all statistical comparisons were performed by either nonparametric Wilcoxon rank-sum or signed-rank tests. SEM was plotted in each figure as an estimate of variation of the mean. Multiple comparisons were addressed with the false discovery rate method when necessary. All statistical analyses were performed using custom MATLAB scripts.

Data accessibility

Data are available on request.

\section{Results}

Inhibitory avoidance assay induced long-term avoidance response while allowing detection of place cell activity across days

To determine the effects of fear acquisition on hippocampal spatial representations, we performed the experiment in a relatively
63

large enclosure $(63 \times 10 \times 30 \mathrm{~cm})$ which both allowed for enough space to assess hippocampal spatial representations and provided ample room for escape runs. The floor consisted of four independently-controllable shock grids. Mice were allowed to freely explore the environment for a period of $10 \mathrm{~min} / \mathrm{d}$ over the course of $6 \mathrm{~d}$. Fear acquisition occurred on day 7 , when ten 0.4 $\mathrm{mA}$ footshocks lasting $2 \mathrm{~s}$ each were delivered at least $21 \mathrm{~s}$ apart. To dissociate the shock from a specific area of the enclosure and thereby avoid a stronger negative association with a particular location in the environment, shocks were distributed across the four separate shock grids, each covering a quarter of the enclosure (see Materials and Methods). The acquisition session was followed by six extinction sessions (Fig. 1A). Total path length decreased (Fig. $1 B$ ), as did speed (not shown, as this is linearly related to path length). However, while the speed was lower in the corner than center quadrants, the speed profiles were maintained across quadrants from baseline to extinction 2. Freezing duration and the number of stretch-attend postures increased for several days following shock delivery (Fig. $1 B, C$ ). It is important to note that, although we did observe a statistically significant increase in fear-induced freeze bouts, the change was smaller than usually observed in traditional fear conditioning assays. This was by design, the dimensions of the full grid enclosure were larger than those of conventional fear conditioning boxes, thus encouraging more exploratory behavior and better spatial sampling.

Throughout each session, calcium activity was recorded by a head-mounted camera (Aharoni and Hoogland, 2019). Individual neurons were identified using CNMFe (Zhou et al., 2018) and coregistered across days using CellReg (Sheintuch et al., 2017; Fig. 1D-F). A total of 4877 neurons were recorded from all mice during all sessions. On average, 219 place cells were recorded during each session from each mouse (for place cell criteria, see Materials and Methods; Fig. $1 G, H)$. We determined that 365 neurons were active across all analyzed sessions, and 2134 neurons were active across half of these sessions. Across contiguous full grid sessions, $12.82 \pm 1.64 \%$ of place cells exhibited stable place fields. (Stable place fields were defined as those which both fulfilled place cell criteria and exhibited a place field center shift of $<5 \mathrm{~cm}$ for neighboring sessions.)

Inhibitory avoidance assay altered place cell firing properties relative to control

We excluded all freeze epochs and restricted our analysis to samples within the same speed range in all sessions (see Materials and Methods) such that mean speed and number of analyzed samples were not significantly different between baseline and 
extinction epochs. Following shock day, place cells exhibited a decrease in mean event rate and place field size (examples in Fig. 2A,B). These changes cannot be explained by unequal sampling of the environment across sessions (Fig. 3; see Materials and Methods). The change from baseline in mean rate and place field size for coregistered cells showed nonsignificant correlations (Fig. 2C), indicating these metrics independently affect cell activity. Moreover, a decrease in place field size did not necessarily lead to an increase in spatial information content; the change in place field size and spatial information content for coregistered cells from baseline to extinction 1 or 2 showed a low correlation, and in the opposite direction this would suggest (Fig. 2D).

Across all recorded place cells, we observed a significant decrease from baseline in mean event rate and place field size (Fig. $2 E)$, a robust effect maintained through extinction and not observed in the control data (Fig. 2G). These changes were accompanied by a significant increase in spatial information content across extinction epochs. While the control data show a modest 0.08 bits/event increase in spatial information content by the second extinction epoch, the corresponding full grid data show a greater increase of 0.13 bits/event (Fig. 2E,G, right). Even when excluding the place cells that show a decrease in place field size from baseline to extinction 1 or 2 , there is still a significant increase in spatial information content for both extinction epochs (Fig. 2F).

To determine whether shock-induced changes in spatial sampling could bias these place cell firing properties, we performed a series of analyses. First, to see whether this effect was because of uneven sampling on the left and right sides of the enclosure, we recalculated these results on a subsampled dataset, such that an equal number of samples was included from the left and right sides of the enclosure per session (see Materials and Methods). The results (Fig. 3A) are nearly indistinguishable from the original findings (Fig. 2E). Moreover, the bimodality coefficient for all preshock sessions (see Materials and Methods; Fig. 3B) did not significantly correlate with our main measures, indicating they are not affected by uneven sampling across the enclosure (Fig. 3C). To address the possibility that reduced spatial sampling could explain this change in place field size (i.e., fewer spatial samples may result in a smaller place field), we calculated the correlation between this measure and the percent occupancy at each place field center, from baseline to Ext 1 epochs. These measures were essentially uncorrelated (Spearman $r=0.085$ ).

We further calculated the change in place field size and spatial information content with the more typical speed range of $>2 \mathrm{~cm} / \mathrm{s}$, and found the results were comparable with those in Figure 2 (Fig. $4 A$ ). Together, these results suggest that, following shock day, place cell ensembles represent mouse position at a finer scale.
Full grid results (velocity threshold $>$ than $2 \mathrm{~cm} / \mathrm{s}$ )
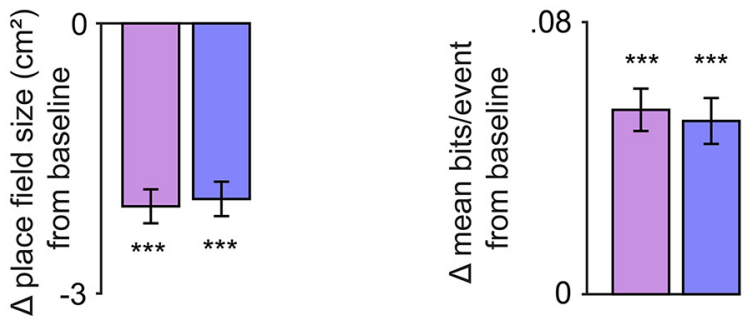

Local grid results (velocity threshold $>$ than $2 \mathrm{~cm} / \mathrm{s}$ )

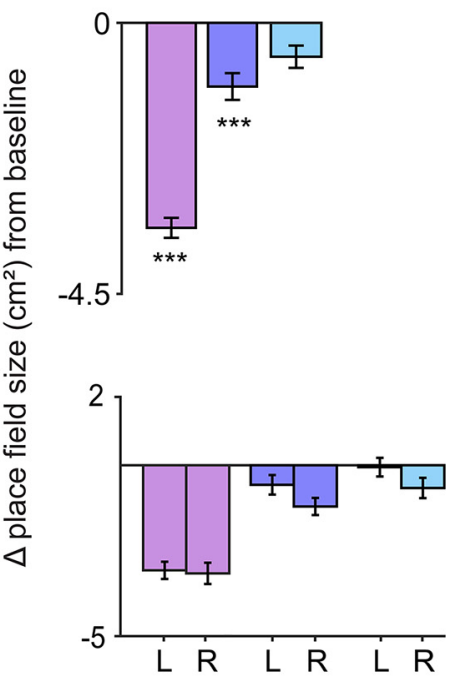

$\triangle B L \&$ Ext. 1

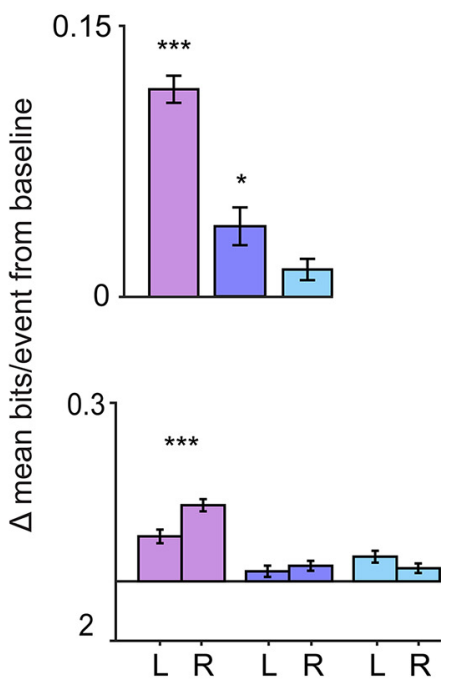

$\triangle B L \&$ Ext. 3
Figure 4. Place cell firing properties calculated using samples for which the speed was higher than $2 \mathrm{~cm} / \mathrm{s}$. $A$, Changes in place cell firing properties from baseline across epochs of the full grid assay. Bars show the changes in firing properties ( \pm 1 SEM) of pooled place cells that coregister between baseline and Ext epochs 1 and 2 ( $n=1620$ 列eline/Ext 1; $n=1535$ baseline/Ext 2). Statistical comparisons are performed by Wilcoxon signed-rank test for grid assay. Bars show the changes in firing properties from baseline of pooled place cells ( $\pm 1 \mathrm{SEM}$ ) that cor(Ext) epochs 1, 2, or 3. Statistical comparisons are performed by properties for place cells with place field centers on the left $(L)$ or right $(R)$ side of the enclosure across 列 baseline/Ext 3). Statistical comparisons made by Wilcoxon rank-sum test for change in left and right sides $(n=24$ per epoch); ${ }^{*} p<0.05,{ }^{* * *} p<0.001$.

\section{Inhibitory avoidance-induced remapping is stronger near the threat}

We next investigated whether these firing changes are modulated by distance to threat. To address this, we modified the previously described assay such that the shock grid was localized to the rightmost side of the enclosure (Fig. 5A; Materials and Methods). In this assay, the large enclosure size was critical, as it allowed us to examine the associations of shock with distinct locations. Again, to control for speed decrease following fear acquisition, only nonfreeze samples within a specific speed range (see Materials and Methods) were included in the analysis, such that there was no significant speed difference preshock and postshock. A total of 6463 neurons were recorded from all mice during all sessions. An average of 175 place cells were recorded during each session from each mouse (for place cell criteria, see Materials and Methods). We determined that 256 
A

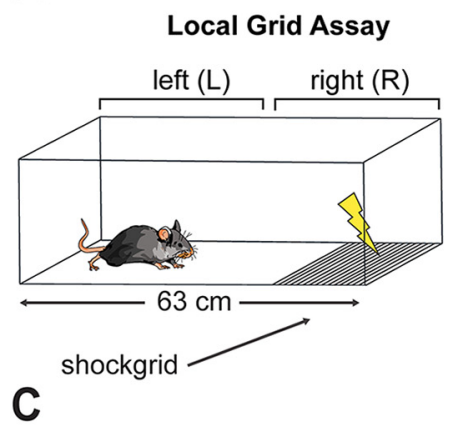

B
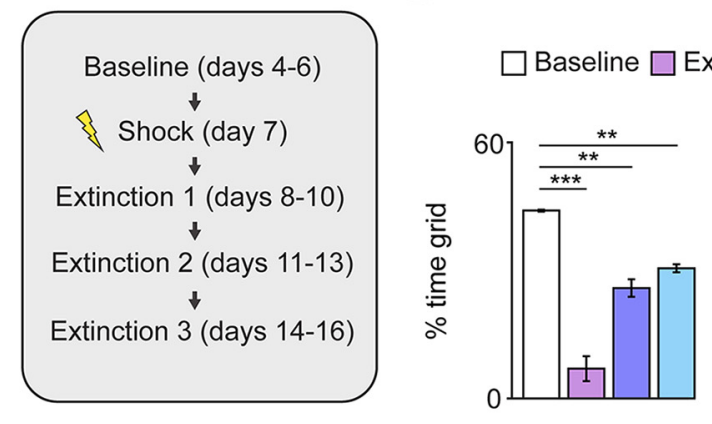

Ext. $1 \square$ Ext. $2 \square$ Ext. 3
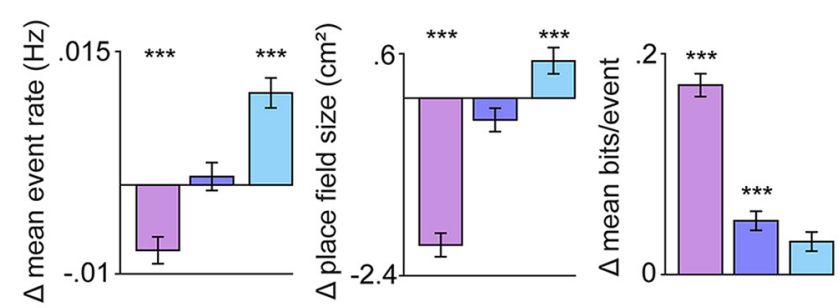
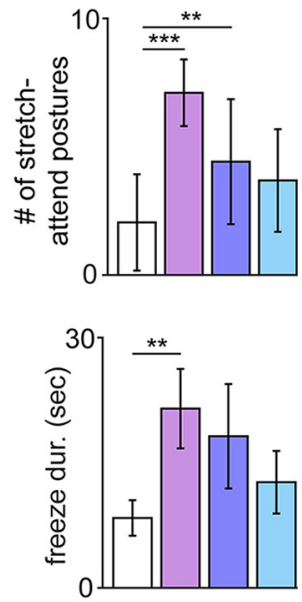

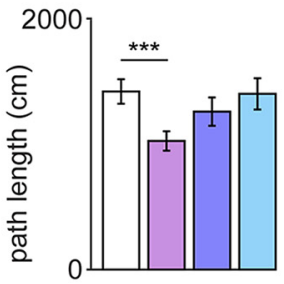

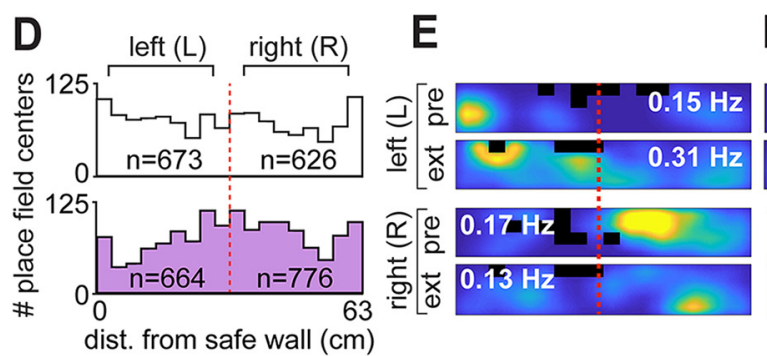

F

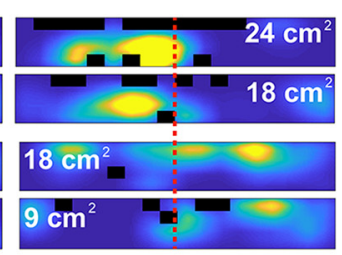

G

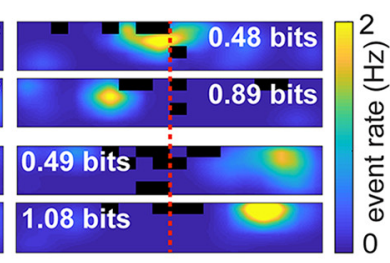

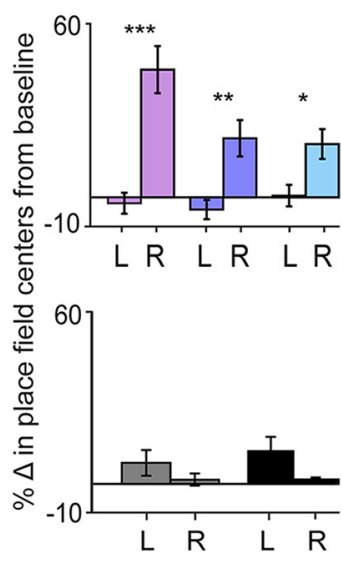

$\triangle \mathrm{BL} \&$ Ext. 1

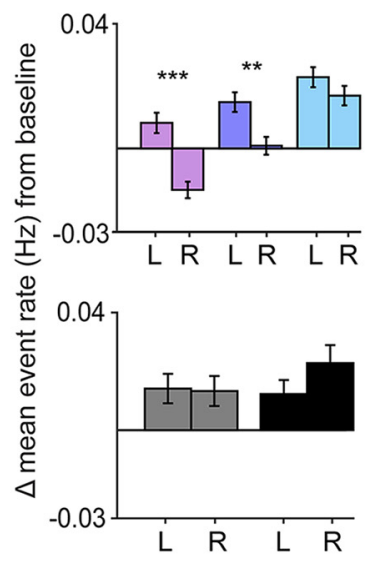

$\triangle$ BL \& Ext. 2
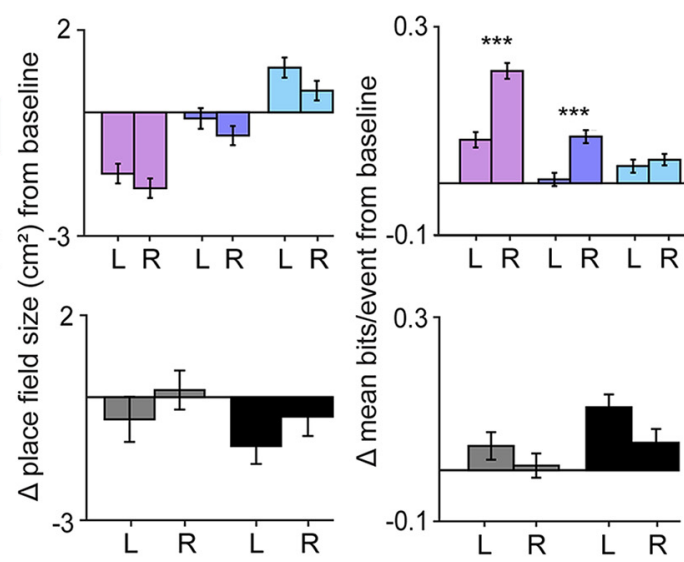

$\triangle$ BL \& Ext. 3

$\Delta$ No shk (4-6) \& No shk (8-10)

$\Delta$ No shk (4-6) \& No shk (11-13)

Figure 5. Remapping effects are more prominent near the shock grid. $\boldsymbol{A}$, Illustration of local grid inhibitory avoidance assay and epoch sequence. $\boldsymbol{B}$, Bars show percent time spent on the shock grid, path length, the number of stretch-attend postures, and freeze duration ( \pm 1 SEM) for recording days $4-16(n=8)$. C, Changes in firing properties of hippocampal CA1 place cells across epochs of local grid assay. Bars show the changes in firing properties from baseline of pooled place cells ( \pm 1 SEM) that coregister between baseline (BL) and extinction (Ext) epochs 1 , 2, or 3 ( $n=1630$ baseline/Ext 1; $n=1591$ baseline/Ext 2; $n=1576$ baseline/Ext 3). Statistical comparisons are performed by Wilcoxon signed-rank test for paired cells at baseline/Ext 1, baseline/Ext 2, and baseline/Ext 3. D, top, Distribution of place field centers for all mice in example baseline and extinction 1 sessions. The number of place fields in the left and right side are annotated inside the histogram. Note that, in extinction 1, there is an increase in the number of fields on the right side, which contains the shock grid. Middle, Bars show the percent change in the number of place field centers ( \pm 1 SEM) from baseline on the left and right sides of the enclosure for Ext epochs 1-3 in shocked mice. Statistical comparisons made by Wilcoxon signed-rank for change in left and right sides ( $n=8$ per epoch). Bottom, Same as middle but for control mice ( $n=4$ per epoch). For $\boldsymbol{E}-\boldsymbol{G}$, the change in firing properties for place cells with place field centers on the left (L) or right (R) side of the enclosure across epochs. Bars show the change in firing properties ( \pm 1 SEM) from baseline of pooled place cells, $L$ and $R$, that coregister between baseline and Ext epochs 1, 2, or 3. $\boldsymbol{E}$, top, Example place fields on the left and right sides of the enclosure that, respectively, increase and decrease mean rate. $\boldsymbol{F}$, top, Example place fields on left and right sides of the enclosure that decrease place field size. $G$, top, Example place fields on the left and right sides of the enclosure that increase information content. Local grid: $n=701$ baseline/Ext 1 left; $n=886$ baseline/Ext 1 right; $n=657$ baseline/Ext 2 left; $n=899$ baseline/Ext 2 right; $n=704$ baseline/Ext 3 left; $n=866$ baseline/Ext 3 right; control: $n=150$ baseline/ Ext 1 left; $n=171$ baseline/Ext 1 right; $n=166$ baseline/Ext 2 left; $n=158$ baseline/Ext 2 right); ${ }^{*} p<0.05,{ }^{* *} p<0.01,{ }^{* * *} p<0.001$. 

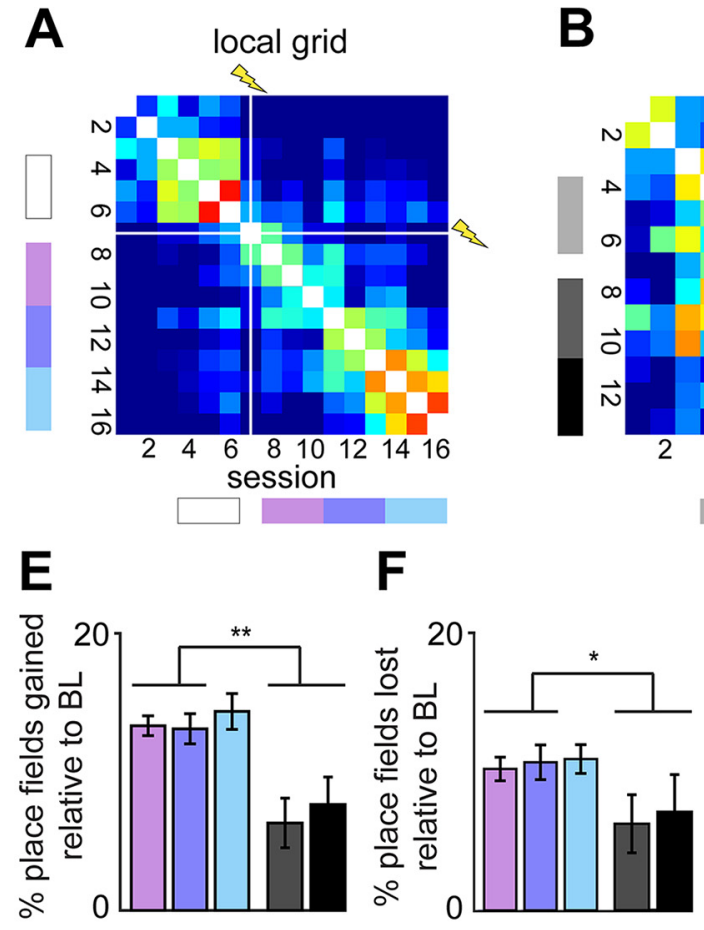

$\square$ Baseline (days 4-6)
No shock (days 4-6)

Ext. 1 (days 8-10)

No shock (days 8-10)
B

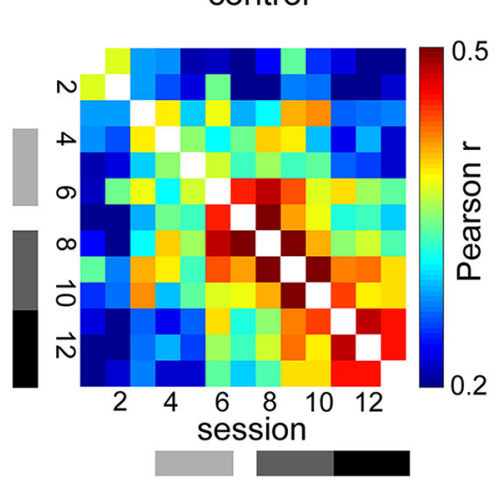

G

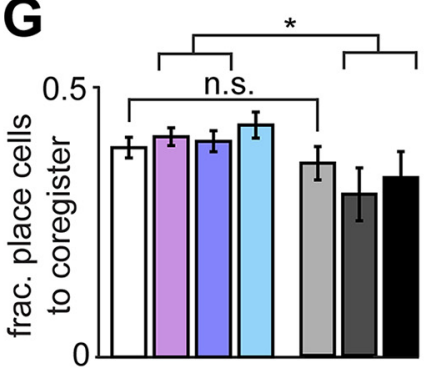

Ext. 2 (days 11-13)

No shock (days 11-13)

C

D
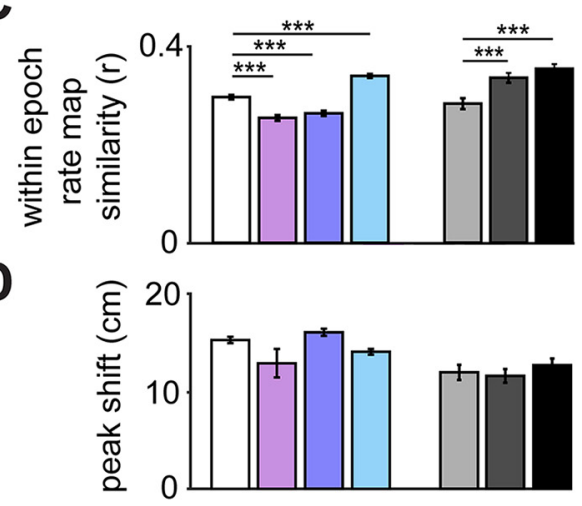

$\mathrm{H}$

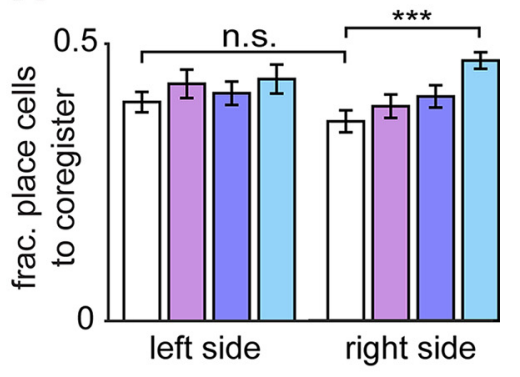

Ext. 3 (days 14-16)

Figure 6. Place field stability measures are affected by the inhibitory avoidance assay. $\boldsymbol{A}$, Correlation matrix showing Pearson $r$ values of the rate maps of coregistered place cells in the local grid and $(\boldsymbol{B})$ control (no-shock) assays across days. For each session correlation pair, only rate map locations with more than five samples in both sessions were used. $\boldsymbol{C}$, Bars show the mean correlation ( \pm 1 SEM) of coregistered cells within neighboring sessions of each epoch, local grid, and control. Statistical comparisons performed by Wilcoxon rank-sum test between days 4 and 6 and all subsequent epochs ( $n=546$, mean cell count per local grid epoch; $n=97$, mean cell count per control epoch). $D$, Bars show the amount of peak shift, defined as the distance ( $\mathrm{cm}$ ) between maximum rate map bins for coregistered neurons in neighboring sessions ( $n=1383$, mean cell count per local grid epoch; $n=295$, mean cell count per control epoch). Only sessions in which $>90 \%$ of the enclosure length was explored were used in this analysis. $\boldsymbol{E}, \boldsymbol{F}$, Bars show the mean percent of place fields gained $(\boldsymbol{E})$ or lost $(\boldsymbol{F})$ in the first session of each extinction epoch, relative to baseline session 6 . Statistical comparisons performed by Wilcoxon rank-sum between days 8 and 13 for local grid and control $(n=16$ local grid sessions, $n=8$ control sessions). $\mathbf{G}$, Bars show the fraction of place cells to coregister for contiguous sessions within each epoch, local grid, and control. Statistical comparisons are performed by Wilcoxon rank-sum between days 4 and 6 and all subsequent epochs ( $n=24$ local grid sessions per epoch, $n=12$ control sessions per epoch). $\boldsymbol{H}$, Bars show the fraction of place cells to coregister within each epoch, separately for the left and right side of the local grid enclosure. Statistical comparisons are performed by Wilcoxon rank-sum between baseline and all subsequent epochs ( $n=24$ local grid left and right sessions per epoch); ${ }^{*} p<0.05,{ }^{* *} p<0.01,{ }^{* * *} p<0.001$, n.s., not significant.

neurons were active across all analyzed sessions, and 2662 neurons were active across half of these sessions. Across contiguous local grid sessions, $15.21 \pm 0.79 \%$ of place cells exhibited stable place fields (for stability criteria, see Materials and Methods).

Fear acquisition in this local grid assay caused a strong aversively-motivated response and a marked change in firing properties. There was a steep reduction in the fraction of time mice spent on the shock grid, as well as an increase in the number of stretch-attend postures and freezing duration (Fig. $5 B$ ). Importantly, these aversively-motivated responses largely extinguished by the end of the experiment, showing that data were collected throughout the extinction process. However, mice continued to explore the non-gridded side of the enclosure. The changes observed in place cell firing properties resembled those in the full grid assay. Mean event rate and place field size decreased for extinction epoch 1 and spatial information content increased for extinction epochs 1-2 (Fig. 5C). These results were maintained for the more typical speed range of $>2 \mathrm{~cm} / \mathrm{s}$ (Fig. $4 B$, top).

As hypothesized, the effects of shock delivery on place cell firing properties were also modulated by distance to threat. In the following analyses, we compare changes in firing properties of cells with place field centers on the left (opposite threat) and right (toward threat) sides of the enclosure (Fig. 5D-G), relative to baseline. Compared with preshock baseline, postshock extinction 1 and extinction 2 sessions showed an increase in place fields on the right side of the context containing the shock grid (Fig. $5 D$ ). In extinction epoch 1, place cells on the left (away from the shock grid) and right sides of the enclosure exhibited a respective increase and decrease of mean event rate from baseline (Fig. $5 E$ ). Both groups showed a similar decrease in place field size (Fig. $5 F)$. Right-side place cells also exhibited a significantly greater increase in spatial information content than left-side place cells (Fig. 5G). These data indicate that several measures changed more markedly near the threatening shock grid than the safer left side. These measures include a decrease in mean rate and increase in spatial information content and number of place field centers, demonstrating stronger remapping near the shock grid. Moreover, these results indicate that the observed changes in place cell firing properties are not mere byproducts of decreased spatial sampling, as a significant change in both place field size and spatial information content was observed for place cells encoding both the left and right sides of the enclosure. As before, these results were comparable for the more typical speed range of $>2 \mathrm{~cm} / \mathrm{s}$ (Fig. $4 B$, bottom). 
A

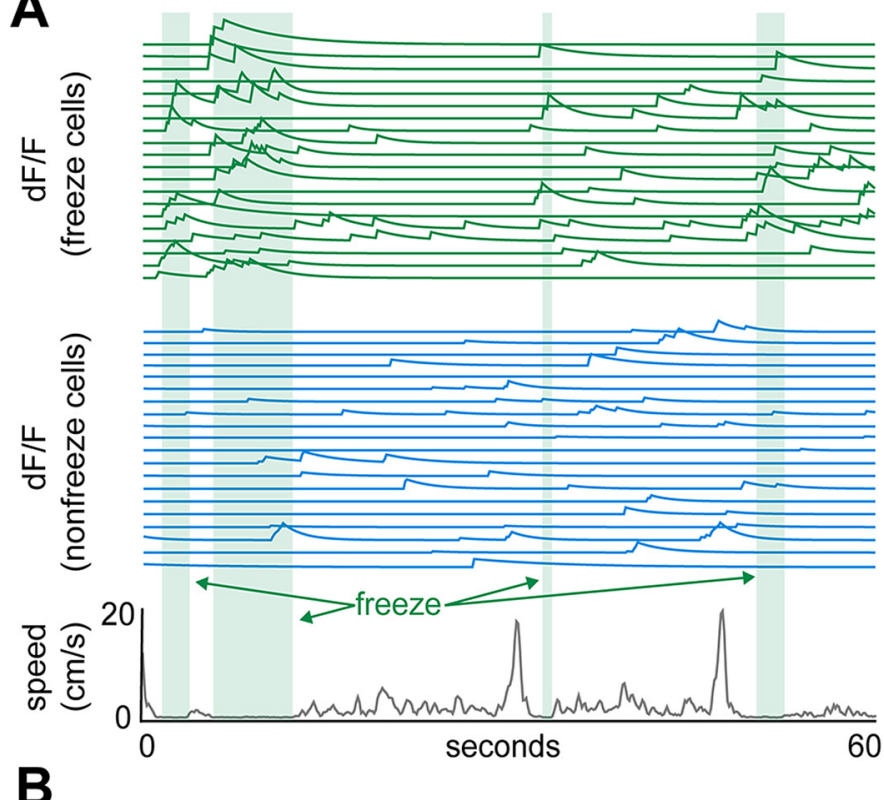

B

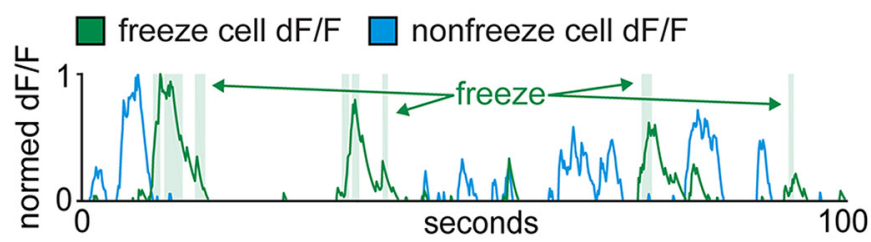

C

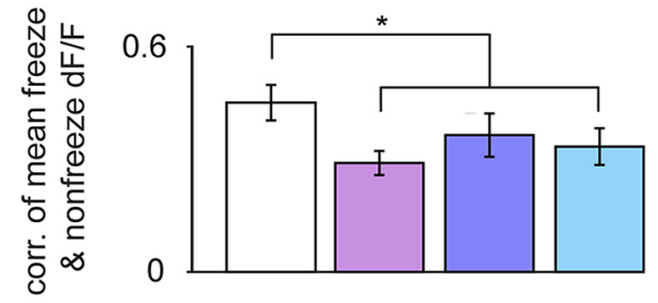

D

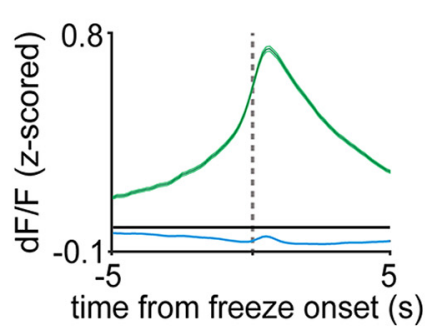

E

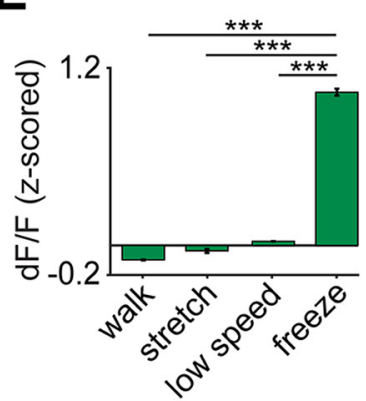

$\mathbf{F}$

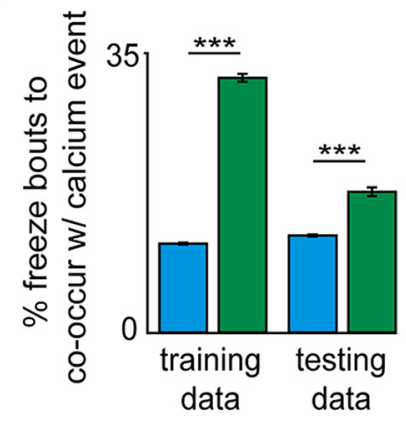

H
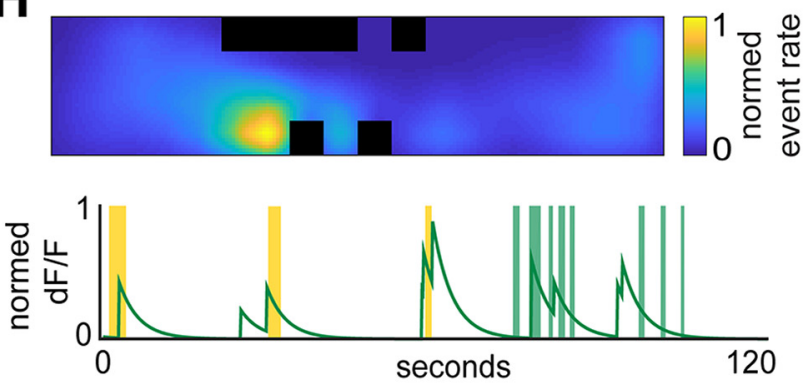

$\square$ freeze behavior $\square$ mouse in place field

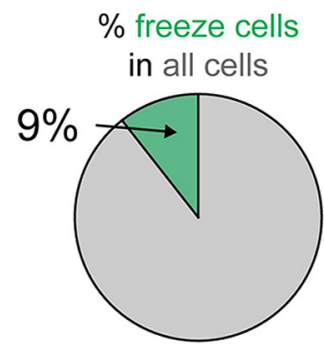

$\%$ place cells in freeze cells

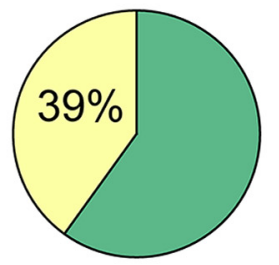

$\%$ freeze cells in place cells

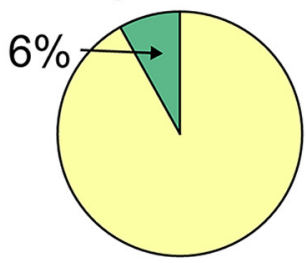

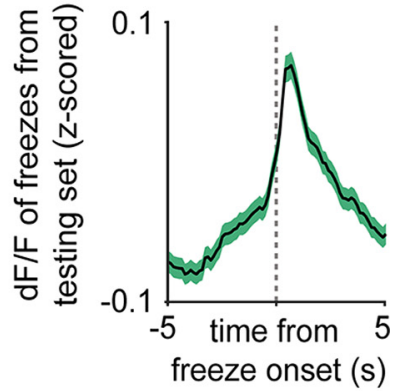

(s) freeze cell $\mathrm{dF} / \mathrm{F}$

Baseline (days 4-6) nonfreeze cell $\mathrm{dF} / \mathrm{F}$ Shock (day 7)
Ext. 1 (days 8-10)

Figure 7. Freeze cell stability and place coding. A, A GLM was used to identify cells that are significantly modulated by freezing (see Materials and Methods). Example recording from an extinction session of (top) individual freeze and nonfreeze cells and (bottom) the corresponding mouse speed. $\boldsymbol{B}$, Example recording of mean z-scored freeze and nonfreeze cell activity, normalized by maximum value. $C$, Bars depict the Spearman correlation $r$ value ( $\pm 1 \mathrm{SEM}$ ) between freeze and nonfreeze cell activity (mean z-scored dF/F of all freeze or nonfreeze cells) for each session. Statistical comparison performed by Wilcoxon rank-sum test between baseline and grouped extinction epochs ( $n=24$ baseline sessions, $n=72$ extinction sessions). $\boldsymbol{D}$, Mean $z$-scored freeze aligned activity for all freeze cells at each behavioral instance, \pm 1 SEM ( $n=41,411$ freeze aligned $\mathrm{dF} / \mathrm{F}$ traces). $E$, Bars show the mean $z$-scored $\mathrm{dF} / \mathrm{F}$ activity during walking, stretch, low speed $(<3 \mathrm{~cm} / \mathrm{s})$ and freeze behavior $(n=2078)$. $\boldsymbol{F}$, A GLM was again used to identify freeze cells, but with the latter half of freeze bouts excluded ("training data" = modeled freeze bouts; "testing data" = excluded freeze bouts). To assure that no single calcium event appeared in both training and testing sets, only freeze bouts from the testing set that were separated by at least $10 \mathrm{~s}$ from those of the training set were used. For each freeze and nonfreeze cell classified in this manner, we quantified the percent of freeze bouts that were accompanied by calcium events per session ( $\pm 3 \mathrm{~s}$ from behavior onset). This was done separately for training and testing sets. Bars show this mean percent measure for ( $\pm 1 \mathrm{SEM}$ ) freeze and nonfreeze cells, across both training and testing sets. $\boldsymbol{G}$, Trace of the mean freeze cell dF/F ( \pm 1 SEM) for freeze bouts from the "testing data" ( $n=13,756$ freeze aligned dF/F traces; for details, see $\boldsymbol{F}$ and Materials and Methods). $\boldsymbol{H}$, Data from an example cell categorized as both freeze and place encoding. Figures show (top) the rate map for an example session and (bottom) activity for the same session with freeze behavior highlighted in green and times in which the mouse traversed the place field in yellow. I, Pie charts show (top left) the percentage of all local grid cells categorized as 
Inhibitory avoidance-induced change in place cell stability

To determine how fear acquisition affected place cell stability over time, we analyzed rate map similarity for place cells in all control and local grid sessions (Fig. 6A-C). The control correlation matrix (Fig. $6 B$ ) shows a gradual increase in correlation over time, as well as a high degree of similarity between temporally distant sessions. In contrast, the local grid correlation matrix (Fig. 6A) shows two large discontinuities: the preshock (days 1-6) and extinction 3 (days 14-16) sessions exhibit high within-epoch stability, while shock and extinction 1-2 (days 7-13) sessions appear relatively unstable. These three distinct periods bear little resemblance with one another. This effect is quantified in Figure $6 C$; while the control grid shows a gradual increase in rate map correlation, the local grid exhibits a significant decrease in this measure for extinction 1-2, rebounding for extinction 3 . Thus, the highest instability in the rate map across days was observed in the sessions immediately following shock, which is when the fear-induced behavioral changes were most prominent (Fig. 5B).

Despite these changing correlation dynamics, there is a fairly consistent amount of peak shift, or the distance separating coregistered place field centers between sessions (Fig. 6D). There is also a significantly higher percentage of place fields gained and lost in the local grid assay than the control assay (Fig. 6E,F), demonstrating that fear acquisition induced a higher turnover of cells. This likely contributes to the instability observed in extinction 1-2. While the fraction of place cells to coregister within epochs is the same for local grid and control sessions at baseline, local grid place cells show a significant increase in this measure over control following shock (Fig. 6G). Moreover, place cells with fields on the right, but not left, side of the enclosure showed increased stability across days following shock (Fig. 6H). Based on this measure, place cells with place field centers nearer the localized threat exhibited greater between-session stability.

\section{Inhibitory avoidance assay differentially affects freeze and nonfreeze cells}

We next investigated whether place cells also encode defensive behavior elicited by this threat, specifically freezing (for behavioral criteria, see Materials and Methods). Freeze duration increased following shock and subsequently decreased over repeated extinction sessions (Fig. 5B). To investigate whether CA1 cells encode freezing behavior, we used a GLM to identify cells that exhibited calcium activity increases during freeze behavior. The model was fit to the activity of each cell, with freeze indices as the predictor variable and freeze coefficient as the measure of fit. Freeze onset times were consistently rolled by multiples of 2-s intervals and a bootstrap distribution built from the resulting GLM coefficients. A

$\leftarrow$

freeze cells per session, (top right) the percentage of all local grid cells categorized as place cells per session, (bottom left) the percentage of these freeze cells categorized as place cells per session, and (bottom right) the percentage of place cells categorized as freeze cells per session $(n=16) ;{ }^{*} p<0.05,{ }^{* * *} p<0.001$. cell was considered a freeze cell if its coefficient exceeded $95 \%$ of the bootstrap coefficient values.

Remarkably, a group of neurons was identified that preferentially fired during freeze behavior, referred to here as "freeze cells." Figure $7 A$ depicts example freeze and nonfreeze cell activity over the course of multiple freeze bouts, as well as mouse speed. For individual sessions, freeze cells preferentially fired during freeze bouts, quite distinct in this regard from nonfreeze activity (Fig. $7 B$ ). Interestingly, the Spearman correlation of freeze and nonfreeze ensemble activity across epochs showed a significant decrease following shock (Fig. 7C). There was no significant difference between calcium decay duration of freeze and nonfreeze cells ( $p=0.25$, Wilcoxon rank-sum test; nonfreeze cell decay duration $3.28 \pm 0.10 \mathrm{~s}$; freeze cell decay duration $3.37 \pm$ $0.09)$.

Freeze cells showed a high degree of behavioral tuning. Mean behavior-aligned freeze cell activity was quite distinct from nonfreeze activity and increased before freeze onset, indicating that these cells are not merely reflecting current freezing (Fig. 7D). Moreover, they exhibited significantly greater activity during freeze behavior than walking, stretch-attend posture, and other low speed moments (Fig. $7 E$ ). To establish the propensity of this group to fire during freeze, as well as address the possibility we had overfit the neural data, we trained a GLM on only the initial half of freeze epochs per session (training data). We then calculated how these freeze cells fired during the latter half of freeze epochs (testing data), excluding those that occurred within $10 \mathrm{~s}$ of the training data (to avoid contamination of the testing set by the slowly decaying calcium signal; see Materials and Methods). We observed that freeze cells were active during a significantly higher percentage of freeze events per session than nonfreeze cells, for both the behavioral instances used to train the model and those reserved for testing (Fig. $7 F, G$ ).

Many of these freeze-activated cells also encoded spatial information (referred to as "freeze-activated place cells"; Fig. 7H). Of all identified cells for each local grid session, $8.7 \pm 0.5 \%$ were categorized as freeze cells (for freeze cell criteria, see Materials and Methods), and 39.2 $\pm 1.4 \%$ of these freeze cells also showed enough spatial selectivity to be classified as place cells on the same session. This is significantly less than the percentage of all cells, $53.4 \%$, that fulfilled place cell criteria $(p<0.001$, Fisher's exact test). For each local grid session, only $6.4 \pm 0.5 \%$ of place cells were also categorized as freeze cells (Fig. 7I). The firing 
A

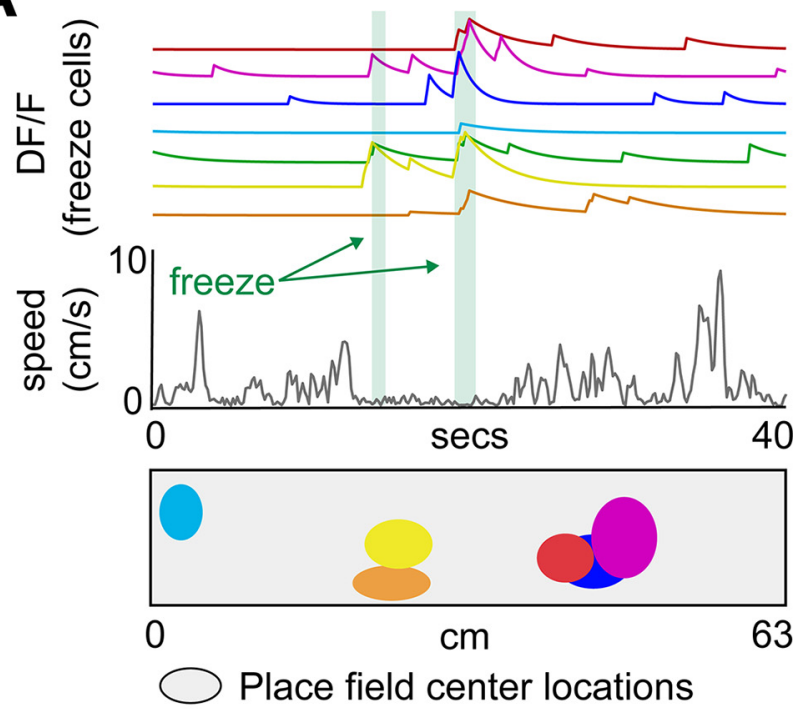

B

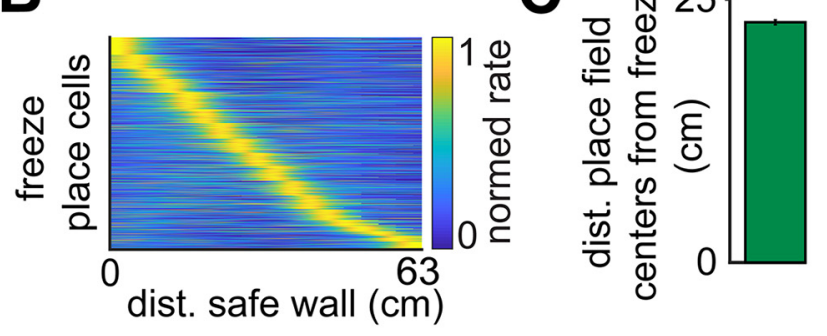

D
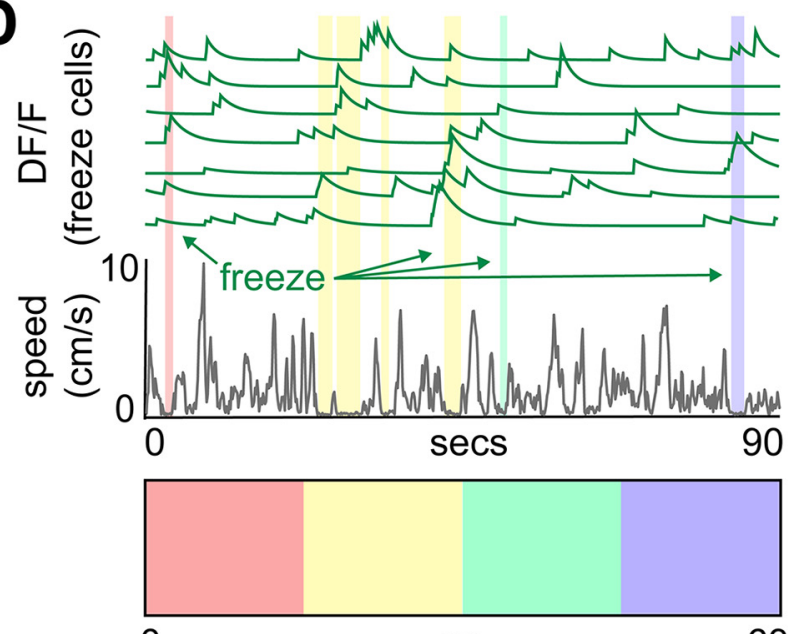

$\mathbf{E}$

0

$\mathrm{cm}$

63

$\%$ freeze cells activated during freeze behavior at multiple locations

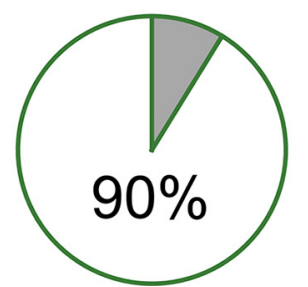

Figure 9. Freeze cells encode freeze behavior rather than freeze location. $\boldsymbol{A}$, top, Example traces of freeze cells in the local grid assay that were also categorized as place cells and (middle) mouse speed. Bottom, Color-coded ovals depict place field centers corresponding to traces (top) of matching color. $\boldsymbol{B}$, Rows depict the rate maps of all freeze-activated place cells in all local grid sessions along the length of the enclosure, sorted by place field center location $(n=1264)$. C, Bar depicts the mean distance ( \pm 1 SEM) of freeze-activated place cell field centers from freeze location for all local grid recording sessions $(n=5268$ freeze- properties of these freeze-activated place cells were similar to those seen in all place cells, and showed similar dynamics over the course of extinction, displaying decreased place field size and increased spatial information following fear acquisition (Fig. 8A$C)$. Because of our use of a synapsin promoter, the genetic identity of this group of freeze cells is unknown.

\section{Freeze cells encode freeze behavior rather than freeze location}

Place field centers of freeze-activated place cells were distributed across the length of the enclosure (Fig. 9A,B) and were located at a significant distance from individual freeze locations (Fig. 9C). For sessions in which freeze bouts occurred at different locations of the enclosure, $90 \%$ of all freeze cells were active across multiple quarters of the enclosure (Fig. 9D,E; see Materials and Methods). These results indicate that freeze cells encode behavior independently from behavior location.

\section{Freeze cell activity is distinct from pSWR activity}

We next determined whether freeze cell activity is distinct from pSWR activity (Foster and Wilson, 2006; Jackson et al., 2006; Diba and Buzsáki, 2007; Davidson et al., 2009; Karlsson and Frank, 2009; Singer and Frank, 2009; Gupta et al., 2010), a phenomenon characterized as $100-250 \mathrm{~Hz}$ ripple oscillations within brief 50- to 400-ms epochs that often describe past or future movement trajectories. Similar to freeze cell activity in the current study, sharp wave ripples are ensemble events known to occur at low speed moments in hippocampal CA1 recordings. We cannot unambiguously identify sharp wave ripple activity, as we did not record local field potentials and will therefore discuss pSWRs. Figure $10 \mathrm{~A}$, top, depicts example cells that are primarily active during either freeze behavior or pSWRs, defined as samples in which the number of active neurons exceeds three standard deviations as previously published (Davidson et al., 2009; Fig. 10A, bottom). In agreement with the literature, the mean pSWR duration ( \pm 1 SEM) was $169.33 \pm 1.33 \mathrm{~ms}$, indicating the majority of these events occur within a single recorded sample $(1 / 7.5=133.33 \mathrm{~ms}$, as the sampling frequency was $7.5 \mathrm{~Hz})$.

Both the mean $\mathrm{z}$-scored calcium activity of all cells and the number of coactive neurons is greater during pSWRs than freeze behavior (Fig. 10B). Freezes rarely accompany pSWR activity, as only $3.53 \pm 0.010 \%$ of all freeze samples occur during pSWRs (Fig. 10C). Also, in agreement with the previous reports (Foster and Wilson, 2006; Joo and Frank, 2018), the gross majority of pSWRs (85.99 $\pm 4.18 \%)$ occurred during non-locomotive, nonfreeze moments (Fig. 10C). Given the difference in behavior and neural activity during freeze bouts and pSWR events, it is likely they are separate phenomena.

\section{Discussion}

\section{Summary of findings}

In the present study, we demonstrate that an inhibitory avoidance assay decreased place field size and increased spatial information content. Interestingly, these effects scaled with threat

activated place cell instances). $\boldsymbol{D}$, top, Example traces of freeze cells activated during freeze bouts at multiple locations within the enclosure and (middle) mouse speed. Color-coded bouts correspond to the enclosure quadrant (bottom) in which they occurred. $\boldsymbol{E}$, For sessions in which freeze bouts occurred in more than one quadrant of the enclosure $(\boldsymbol{D}$, bottom), $90 \%$ of all freeze cells were active during freeze bouts across multiple quadrants $(n=309$ freeze cell instances; see Materials and Methods). 
A

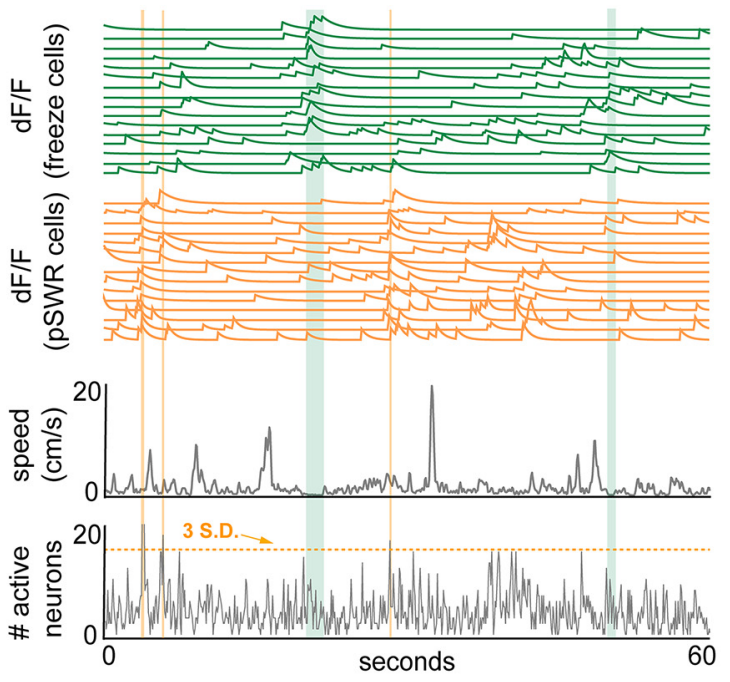

B

freeze bouts
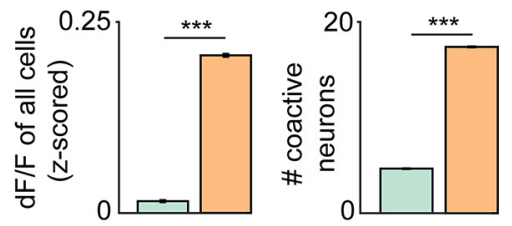

C

$\%$ pSWR events during speed ranges

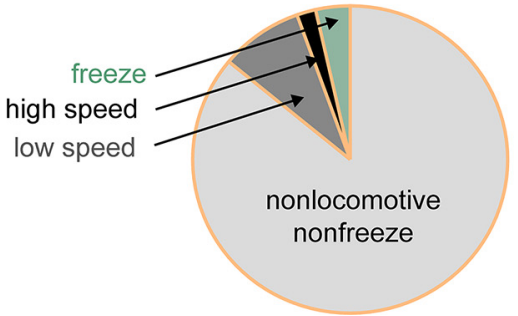

Figure 10. Freeze cell activity is distinct from pSWR activity. A, Example recording of (top) freeze cells and cells that contribute to pSWR activity, (middle) the corresponding mouse speed, and (bottom) the number of active neurons per sample, with the 3-SD threshold shown for pSWR categorization. $\boldsymbol{B}$, Bars compare $\mathrm{dF} / \mathrm{F}$ ( \pm 1 SEM) of all cells (left) and the number of coactive neurons (right) during all freeze bouts and pSWR events ( $n=1616$ freeze bouts, $n=8378$ pSWR events). C, Pie chart depicts the fraction of pSWR events that occurred during freeze bouts $(3.53 \pm 0.99 \%)$, periods of low speed locomotion $(<4 \mathrm{~cm} / \mathrm{s} ; 8.52 \pm 3.54 \%)$, high speed $(>4 \mathrm{~cm} / \mathrm{s} ; 1.96 \pm 1.27 \%)$, and non-locomotive, nonfreeze samples (85.99 $\pm 4.18 \%$; $n=8)$; ${ }^{* * *} p<0.001$

proximity, suggesting an enhancement of threat encoding following shock. This is the first study to investigate the long-term effects of fear acquisition on place cell remapping. We thus describe the effects of fear acquisition and extinction on key stability metrics, finding that the inhibitory avoidance assay induced a period of decreased between-session spatial correlation and greater place cell turnover, followed by a period of enhanced stability that appeared in parallel with the extinction of fear behaviors. Finally, we identify a novel group of freeze-activated neurons that encode both freeze behavior and, in some instances, spatial location with a canonical place field independent of the freezing location.

\section{Comparison with prior fear-induced remapping reports}

Following the delivery of shock in both full and local grid assays, place cells responded with a decrease in mean event rate and place field size and an increase in information content, changes that, altogether, suggest an enhanced encoding of mouse position. This shift makes intuitive sense, as the coding of space on a finer scale would allow for improved threat avoidance.

These findings expand on previous studies, demonstrating that changes in the emotional valence of a context can alter place cell firing properties. Before discussing this body of work, it is important to note that none of these remapping studies implemented a classical contextual fear conditioning protocol with unavoidable and inescapable foot shock as US (unconditioned stimulus). Moita et al. (2004) perhaps came closest, delivering an inescapable and unavoidable shock to the eyelid. In the case of Wang et al. (2012, 2015), the US was a coyote-urine soaked cotton square, while Kim et al. (2015) implemented a surging robotic predator. In these instances, while the US was unavoidable, it was, to some extent, escapable. The same is true for the presently described full and local grid assays. While classical foot shock served as the US, this shock was avoidable/escapable so that spatial sampling would not be reduced by excessively high levels of freezing. Thus, we use "inhibitory avoidance" rather than "fear conditioning" to describe the present assays. Despite these variations, the discussed assays all reported some form of contextual fear-induced behavioral change and some degree of hippocampal remapping. Therefore, they are relevant to the discussion and, while not adhering to a precise classical fear conditioning protocol, are related under the broader category of fearinduced remapping studies.

In contrast to our findings, studies using shock (Moita et al., 2004; Wu et al., 2017) and predator odor (Wang et al., 2015) as the US showed a null effect of contextual fear on information content; further, Moita et al. (2004) reported an increase in place field size following shock. These discrepancies could be explained by any number of experimental differences including the explicit location for shock in the present study, species (mice vs rats), the amount of preexposure to the experimental context, the strength of the US, as well as the behavioral motivation (selfmotivated exploration vs foraging for food pellet), although the relative contributions of these factors remain unclear.

We further demonstrated that fear-induced remapping scaled with distance from the shock grid. Only one previous study (Kim et al., 2015), to our knowledge, has investigated place cell remapping at variable distance to threat; exposing a mouse to a surging "predatory" robot induced a significant change in rate map correlation nearest the threat. This remapping was not stable and reverted following removal of the robotic predator. We similarly observed fear-induced spatial remapping, although the changes did not revert to baseline in the post-threat sessions (Fig. 6A) and were accompanied by increases in both information content and place field count and a decrease in place field size that were more pronounced near the grid (Fig. 5). Thus, remapping in the current report was longer lasting and involved changes in several more place cell features than robotic predator-induced remapping. While the predatory robot was aversive, shock-induced pain is a considerably stronger and more salient US, a difference that could explain the greater degree of remapping we observed.

We also described, to an unprecedented degree, the longterm effects of fear acquisition on place cell stability. Previous 
studies were limited by their use of implanted electrodes, which can shift over time, making it difficult to record from the same neuron for many days. Addressing this issue with chronic calcium imaging technology, we initially examined one key stability metric, the correlation of place cell rate maps over time. This provided a novel look at the changing hippocampal spatial code from preshock to postshock epochs. The control data exhibit a gradual increase in place cell rate map correlation across both contiguous and temporally distant sessions (Fig. 6B), indicating that the hippocampal spatial code grows more stable with repeated exposure to an unchanging environment (Gonzalez et al., 2019). In contrast, the local grid correlation matrix (Fig. 6A) is composed of three qualitatively dissimilar periods; the preshock and extinction three epochs are both characterized by high within-epoch stability, although bear little resemblance to one another, while the extinction 1-2 epochs show significantly less within-epoch stability than baseline (Fig. 6C). Thus, there is higher instability in the spatial code during sessions immediately following shock exposure, which is also when the most robust fear-induced behavior was observed (Fig. 5B). During the final sessions when this fear-induced behavior had largely extinguished (Fig. 5B) the hippocampal representation converged onto a new stable representation (Fig. 6A), in agreement with the view that extinction is new learning rather than erasure of the fear memory. These data indicate that fear behavior is correlated with decreased spatial map stability across days.

This finding agrees in part with previous work (Wang et al., 2015), showing that exposure to a mild US, predator urine odor, induced spatial remapping. These postextinction rate maps (24 and $48 \mathrm{~h}$ postextinction) were also less correlated with one another than observed in the control data, suggesting that the inhibitory avoidance assay would induce a chronic decrease in rate map stability. However, in light of the present results, the continued instability reported by Wang et al. was likely because of incomplete fear extinction. Given the extended recording duration afforded by calcium imaging, we were able to show that, following a longer extinction period, rate maps eventually converge to a novel, more stable spatial representation. In fact, based on changes to other stability metrics, such as the significant increase in the fraction of place cells to coregister between sessions (Fig. $6 G, H)$, this representation grows more stable by late extinction than that of the control group.

\section{Characterization of a novel freeze-activated population in the hippocampus}

While previous studies have analyzed the effects of an US on place cell remapping, they did not consider place cell activity during fear-induced freezing behavior. It was, therefore, unknown whether hippocampal CA1 neurons might play an additional role in coding fear-induced aspects of behavior. Here we have identified a novel group of cells that is preferentially active during freezing bouts and not during other low speed moments or behaviors (Fig. 7E). Perhaps unsurprisingly, this cell group also shows some degree of spatial selectivity, as $39.2 \pm$ $1.4 \%$ of all freeze cells also fulfilled place cell criteria (Fig. 7I).

This conjunction of freeze and place coding suggested a possible confound, perhaps these freeze cells were encoding a stereotyped freeze location rather than freeze behavior. Previous work has, in fact, characterized a group of hippocampal place cells that code for spatial location during non-fear related immobility (Kay et al., 2016), a behavior distinct from defensive freezing. In a series of analyses, we show that the presently-described freeze cells encode freeze behavior rather than freeze location. (1) Freeze- activated place cells recruited by single freeze events shared no common spatial representation, as their place fields were widely scattered across the enclosure (Fig. 9A-C). (2) The majority of freeze cells $(90 \%)$ were active during freeze bouts at multiple locations within the enclosure (Fig. $9 D, E$ ), providing strong evidence that freeze-activated place cells independently represent spatial location and freeze behavior. (3) Regarding the cell group reported by Kay et al., the freeze cells of the present study are located in the CA1 region of the hippocampus while those encoding spatial location during immobility are specific to the CA2 region. Altogether, given these findings, it is clear these freeze cells independently encode behavior and spatial location and are distinct from the reported population in CA2.

In summary, we build on previous pioneering research to show that, in addition to encoding the geometry of an environment, hippocampal place cells encode space to a finer degree following fear acquisition, an effect that scales with distance to threat. Additionally, a previously unrecognized group of hippocampal CA1 cells robustly encodes freeze behavior. Together, these results enrich our understanding of the long-term remapping dynamics following a fearful stimulus, as well as the role the hippocampus may play in related defensive behaviors.

\section{References}

Aharoni D, Hoogland TM (2019) Circuit investigations with open-source miniaturized microscopes: past, present and future. Front Cell Neurosci 13:141.

Aharoni D, Khakh BS, Silva AJ, Golshani P (2019) All the light that we can see: a new era in miniaturized microscopy. Nat Methods 16:11-13.

Alme CB, Miao C, Jezek K, Treves A, Moser EI, Moser MB (2014) Place cells in the hippocampus: eleven maps for eleven rooms. Proc Natl Acad Sci USA 111:18428-18435.

Anderson MI, Jeffery KJ (2003) Heterogeneous modulation of place cell firing by changes in context. J Neurosci 23:8827-8835.

Cai DJ, Aharoni D, Shuman T, Shobe J, Biane J, Song W, Wei B, Veshkini M, La-Vu M, Lou J, Flores SE, Kim I, Sano Y, Zhou M, Baumgaertel K, Lavi A, Kamata M, Tuszynski M, Mayford M, Golshani P, et al. (2016) A shared neural ensemble links distinct contextual memories encoded close in time. Nature 534:115-118.

Davidson TJ, Kloosterman F, Wilson MA (2009) Hippocampal replay of extended experience. Neuron 63:497-507.

Diba K, Buzsáki G (2007) Forward and reverse hippocampal place-cell sequences during ripples. Nat Neurosci 10:1241-1242.

Ellison AM (1987) Effect of seed dimorphism on the density-dependent dynamics of experimental populations of Atriplex triangularis (chenopodiaceae). Am J Bot 74:1280-1288.

Engelhard B, Finkelstein J, Cox J, Fleming W, Jang HJ, Ornelas S, Koay SA, Thiberge SY, Daw ND, Tank DW, Witten IB (2019) Specialized coding of sensory, motor and cognitive variables in VTA dopamine neurons. Nature 570:509-513.

Foster DJ, Wilson MA (2006) Reverse replay of behavioural sequences in hippocampal place cells during the awake state. Nature 440:680-683.

Frankland PW, Cestari V, Filipkowski RK, McDonald RJ, Silva AJ (1998) The dorsal hippocampus is essential for context discrimination but not for contextual conditioning. Behav Neurosci 112:863-874.

Friedrich J, Zhou P, Paninski L (2017) Fast online deconvolution of calcium imaging data. PLoS Comput Biol 13:e1005423.

Gonzalez WG, Zhang H, Harutyunyan A, Lois C (2019) Persistence of neuronal representations through time and damage in the hippocampus. Science 365:821-825.

Gupta AS, van der Meer MAA, Touretzky DS, David Redish A (2010) Hippocampal replay is not a simple function of experience. Neuron 65:695-705.

Jackson JC, Johnson A, Redish AD (2006) Hippocampal sharp waves and reactivation during awake states depend on repeated sequential experience. J Neurosci 26:12415-12426. 
Joo HR, Frank LM (2018) The hippocampal sharp wave-ripple in memory retrieval for immediate use and consolidation. Nat Rev Neurosci 19:744757.

Karlsson MP, Frank LM (2009) Awake replay of remote experiences in the hippocampus. Nat Neurosci 12:913-918.

Kay K, Sosa M, Chung JE, Karlsson MP, Larkin MC, Frank LM (2016) A hippocampal network for spatial coding during immobility and sleep. Nature 531:185-190.

Kim EJ, Park M, Kong M-S, Park SG, Cho J, Kim JJ (2015) Alterations of hippocampal place cells in foraging rats facing a "predatory" threat. Curr Biol 25:1362-1367.

Kinsky NR, Sullivan DW, Mau W, Hasselmo ME, Eichenbaum HB (2018) Hippocampal place fields maintain a coherent and flexible map across long timescales. Curr Biol 28:3578-3588.e6.

Kubie JL, Muller RU (1991) Multiple representations in the hippocampus. Hippocampus 1:240-242.

Latuske P, Kornienko O, Kohler L, Allen K (2018) Hippocampal remapping and its entorhinal origin. Front Behav Neurosci 11:253.

Leutgeb JK, Leutgeb S, Treves A, Meyer R, Barnes CA, McNaughton BL, Moser MB, Moser EI (2005a) Progressive transformation of hippocampal neuronal representations in "morphed" environments. Neuron 48:345358.

Leutgeb S, Leutgeb JK, Barnes CA, Moser EI, McNaughton BL, Moser MB (2005b) Independent codes for spatial and episodic memory in hippocampal neuronal ensembles. Science 309:619-623.

Mathis A, Mamidanna P, Cury KM, Abe T, Murthy VN, Mathis MW, Bethge M (2018) DeepLabCut: markerless pose estimation of user-defined body parts with deep learning. Nat Neurosci 21:1281-1289.

Moita MAP, Rosis S, Zhou Y, LeDoux JE, Blair HT (2004) Putting fear in its place: remapping of hippocampal place cells during fear conditioning. J Neurosci 24:7015-7023.

Muller RU, Kubie JL (1987) The effects of changes in the environment on the spatial firing of hippocampal complex-spike cells. J Neurosci 7:19511968.

O'Keefe J, Dostrovsky J (1971) The hippocampus as a spatial map. Preliminary evidence from unit activity in the freely-moving rat. Brain Res 34:171-175.
O’Keefe J, Conway DH (1978) Hippocampal place units in the freely moving rat: why they fire where they fire. Exp Brain Res 31:573-590.

Phillips RG, LeDoux JE (1994) Lesions of the dorsal hippocampal formation interfere with background but not foreground contextual fear conditioning. Learn Mem 1:34-44.

Pinto L, Rajan K, DePasquale B, Thiberge SY, Tank DW, Brody CD (2019) Task-dependent changes in the large-scale dynamics and necessity of cortical regions. Neuron 104:810-824.e9.

Sheintuch L, Rubin A, Brande-Eilat N, Geva N, Sadeh N, Pinchasof O, Ziv Y (2017) Tracking the same neurons across multiple days in Ca2 + imaging data. Cell Reports 21:1102-1115.

Singer AC, Frank LM (2009) Rewarded outcomes enhance reactivation of experience in the hippocampus. Neuron 64:910-921.

Skaggs WE, McNaughton BL, Gothard KM (1993) An information-theoretic approach to deciphering the hippocampal code. Adv Neural Inf Process Syst 5:1030-1037.

Thompson LT, Best PJ (1989) Place cells and silent cells in the hippocampus of freely-behaving rats. J Neurosci 9:2382-2390.

Thompson LT, Best PJ (1990) Long-term stability of the place-field activity of single units recorded from the dorsal hippocampus of freely behaving rats. Brain Res 509:299-308.

Wang ME, Wann EG, Yuan RK, Ramos Álvarez MM, Stead SM, Muzzio IA (2012) Long-term stabilization of place cell remapping produced by a fearful experience. J Neurosci 32:15802-15814.

Wang ME, Yuan RK, Keinath AT, Ramos Álvarez MM, Muzzio IA (2015) Extinction of learned fear induces hippocampal place cell remapping. J Neurosci 35:9122-9136.

Wilson MA, McNaughton BL (1993) Dynamics of the hippocampal ensemble code for space. Science 261:1055-1058.

Wood ER, Dudchenko PA, Eichenbaum H (1999) The global record of memory in hippocampal neuronal activity. Nature 397:613-616.

Wu CT, Haggerty D, Kemere C, Ji D (2017) Hippocampal awake replay in fear memory retrieval. Nat Neurosci 20:571-580.

Zhou P, Resendez SL, Rodriguez-Romaguera J, Jimenez JC, Neufeld SQ, Giovannucci A, Friedrich J, Pnevmatikakis EA, Stuber GD, Hen R, Kheirbek MA, Sabatini BL, Kass RE, Paninski L (2018) Efficient and accurate extraction of in vivo calcium signals from microendoscopic video data. Elife 7:e28728. 